\title{
Maize protein phosphatase gene family: identification and molecular characterization
}

\author{
Kaifa Wei ${ }^{*}$ and Si Pan
}

\begin{abstract}
Background: Protein phosphatases (PPS) play critical roles in various cellular processes through the reversible protein phosphorylation that dictates many signal transduction pathways among organisms. Recently, PPs in Arabidopsis and rice have been identified, while the whole complement of PPs in maize is yet to be reported.

Results: In this study, we have identified 159 PP-encoding genes in the maize genome. Phylogenetic analyses categorized the ZmPP gene family into 3 classes (PP2C, PTP, and PP2A) with considerable conservation among classes. Similar intron/exon structural patterns were observed in the same classes. Moreover, detailed gene structures and duplicative events were then researched. The expression profiles of ZmPPs under different developmental stages and abiotic stresses (including salt, drought, and cold) were analyzed using microarray and RNA-seq data. A total of 152 members were detected in 18 different tissues representing distinct stages of maize plant developments. Under salt stress, one gene was significantly up-expressed in seed root (SR) and one gene was down-expressed in primary root (PR) and crown root (CR), respectively. As for drought stress condition, 13 genes were found to be differentially expressed in leaf, out of which 10 were up-regulated and 3 exhibited down-regulation. Additionally, 13 up-regulated and 3 down-regulated genes were found in cold-tolerant line ETH-DH7. Furthermore, real-time PCR was used to confirm the expression patterns of ZmPPs.
\end{abstract}

Conclusions: Our results provide new insights into the phylogenetic relationships and characteristic functions of maize PPs and will be useful in studies aimed at revealing the global regulatory network in maize abiotic stress responses, thereby contributing to the maize molecular breeding with enhanced quality traits.

\section{Background}

The reversible protein phosphorylation is a fundamental mechanism that modulates many cellular functions including regulating developmental events and perceiving environmental stimuli [1]. During phosphorylation, protein kinases (PKs) mainly phosphorylate serine (Ser), threonine (Thr) and tyrosine (Tyr) residues, while protein phosphatases (PPs) can reverse this process by removing the phosphate group. Based on substrate specificities, PPs can be categorized into two groups: Ser/Thr and Tyr phosphatases. Recently, 1241 maize PK-encoding genes have been identified, with the data suggesting that maize PKs were implicated in diverse biological processes, such as developmental control and drought stress [2,3]. The development of a flowering plant both at the cellular and organismal level is a highly complex phenomenon. In spite of the

\footnotetext{
*Correspondence: kaifa-wei@163.com
School of Biological Sciences and Biotechnology, Minnan Normal University,

* Correspondence: kaifa-wei@163.com
School of Biological Sciences and Biotechnology, Minnan Normal University, *Correspondence: kaifa-wei@163.com
School of Biological Sciences and Biotechnology, Minnan Normal University,
Zhangzhou 363000, China
}

apparent autonomy, roots, leaves, and other structures perceive external signals and use different signaling mechanisms to respond. Like functions of mammalian PP genes [4], plant PPs are expected to be the key components in signal transduction networks at distinct stages of plant development and in response to multiple abiotic signals. Specific inhibitor (okadaic acid and calyculin A) of Ser/ Thr phosphatases PP1 and 2A-type protein phosphatase (PP2A) arrest root hair growth in a tiny range, and severely affect the shape of cells within the elongation zone and inhibit root growth. In addition, PP2A was associated with controlling microtubules length [5]. As reported in Arabidopsis, PLL4 and PLL5 (POL-like gene) regulate leaf development, but having no detectable functions within the meristem [6]. Flowering time is a major adaptive trait and an important selection criterion in plant breeding. Kim et al. [7] showed that a self-regulatory phytochrome kinase-phosphatase coupling is a key signaling component in the photoperiodic control of flowering in Arabidopsis. 
Plants as sessile organisms are constantly challenged by a wide range of abiotic stresses such as salinity, drought, and cold. However, stresses are not necessarily a problem for plants because they have used several strategies to avoid or reduce the possible damage. Drought limits plant growth and result in a drastic decline in the photosynthetic yield due to osmotic stress-imposed constraint. In order to cope with drought stress, plants utilize various mechanisms, known as drought escape, drought avoidance and drought tolerance [8]. Previous study showed that Group A PP2Cs were negative regulators in ABA signaling pathway and acted as key regulators of desiccation tolerance in land plants [9]. Salinity is one of the most severe environmental factors that greatly impacts plant development and restricts crop production. Furthermore, salt stress may cause water deficit, ion toxicity, nutrient imbalance, and oxidative stress. Interestingly, salinity tolerance is more likely to be a complex multigenic trait involving responses to cellular osmotic and ionic stresses. In Arabidopsis, over-expression of AtPP2CG1 increased salt tolerance, whereas its loss of function impaired salt tolerance [10]. Additionally, cold stress is another key environmental factor that limits the geographic distribution of plants. Cold acclimation has been described as the process in which plants adjust their metabolism during cold treatment. In spite of the fact that maize is generally sensitive to low temperatures [11], the extent of cold sensitivity is different within the maize germplasm. The cold signal is initially perceived by plasma membrane with the help of specific $\mathrm{Ca}^{2+}$ channel proteins, membrane histidine kinases and some unknown sensors, which then activate the sophisticated cold-responsive signaling pathways in concert with plant hormone signaling, the circadian clock, and the developmental transition to flowering. Two PP2Cs, ABI1 and AtPP2CA, which were reported as negative regulators in ABA signaling, exhibited differences in their tissue-specific expressions as well as in temporal induction in response to chilling [12]. To improve the crop yields under abiotic stress conditions, it is crucial to understand the fundamental molecular mechanisms behind stress tolerance in plants.

One hundred twelve and one hundred thirty-two candidate PP genes were identified in Arabidopsis and rice, respectively [13,14]. In Arabidopsis, Group A PP2Cs, $A B I 1$ and $A B I 2$, are negative regulators of $A B A$ signaling, whereas Group B are characterized to regulate mitogen-activated protein kinase (MAPK) signaling cascades. POL-type phosphatases, members of Group C $\mathrm{PP} 2 \mathrm{C}$, are involved in flower development. Independently, another PP2C member, kinase-associated protein phosphatase (KAPP) is a singleton that regulates receptor-like kinases [15]. PTPs are critical partners for tyrosinespecific kinases in regulating the tyrosine phosphorylation status of many proteins. Tyrosine phosphorylation plays a role in MAPK signaling cascades. In particular, the level of tyrosine phosphorylation is determined by the balanced activity of protein tyrosine kinases (PTKs) and PTPs. Moreover, previous study showed that tyrosine phosphorylation is involved in phytohormone responses [16]. Compared with plants, other genomes which lack tyrosine kinases also have very few PTPs. Hence, identification of PTPs provides a stepping stone to better understanding of the functional significance of tyrosine phosphorylation in higher plants.

Although recent advances in higher plants, only a small portion of the members in this family have been found to be components of signaling pathway in maize. This include, $\mathrm{ZmPP} 2 \mathrm{Ca}$ ( $\mathrm{ZmPP}$ ), which is involved in the signal transduction in regulating response to drought stress [17]; ZmPP2C2 (ZmPP159), which plays a positive role in tobacco cold resistance [18]; ZmPP2C (ZmPP76), which is implicated in Arabidopsis stress signal transduction [19]; ZMPP2 (ZmPP138), which was selected as a candidate for the catalytic subunit of phospho-pyruvate dehydrogenase phosphatase (PDP) [20]; ZmRIP1 (ZmPP1), which acts as a chloroplast-to-nucleus signaling messenger, and is confirmed to function in maize redox signaling [21]; ZmPP1 (ZmPP65), whose function and regulation might be very similar to that of mammalian PPs [22].

In the post-genome era, the whole-genome sequencing, together with the global transcriptome profiling, such as microarray and RNA-seq, offer the opportunity to identify diverse gene families and to unravel the functions of genes involved in processes such as developmental regulation, disease resistance and abiotic stress. Maize is not only one of the most important food crops of the world, but also a model plant for study of the genetics in monocotyledons. In this study, we identified the full complement of PP genes in maize genome for evolutionary and functional analyses, which would help crop breeders to develop improved varieties.

\section{Methods}

\section{Identification and characterization of $\mathrm{ZmPPs}$}

The maize protein sequences were downloaded from the Maize Genome Sequence Project (http://ftp.maizesequence. org/release-5b/filtered-set/). To uncover all the members of PPs in maize, the predicted phosphatase sequences from Arabidopsis thaliana and Oryza sativa were used as query sequences to search against maize protein database using HMMER 3.0 software [23]. Firstly, we used hmmbuild tool from HMMER to build the PP hidden Markov model (HMM) profile from the alignments of the known PP sequences. Secondly, hmmsearch program was separately applied to a search of all the protein sequences in maize protein database with the Pfam PP profiles (PF00481: PP2C, PF01451: LMWPc, PF00782: DSPc, PF00102: Y phosphatase) and PP HMM profile, 
and the E-value cut-off was set to 1 . Subsequently, each protein sequence was subjected to SMART (http://smart. embl-heidelberg.de/) and Pfam (http://pfam.sanger.ac.uk/) databases to ensure the presence of the catalytic domain. Proteins without a phosphatase catalytic domain were removed from the dataset. After eliminating the incomplete sequences manually, 159 sequences remained were finally identified as ZmPPs and renamed based on their loci on chromosomes. The subcellular localization of ZmPPs was predicted by WoLF PSORT (http://wolfpsort.org/). In addition, the molecular weights (MWs) and isoelectric points (pIs) of ZmPPs were predicted by ExPASy Server tool (http://web.expasy.org/compute_pi/).

\section{Phylogenetic analysis and classification of the ZmPP gene family}

Catalytic domains of ZmPPs were used for multiple alignments with the aid of MEGA5 software (http://www. megasoftware.net/) by employing ClustalW as the algorithm. Phylogenetic trees were constructed by neighborjoining (NJ) algorithm of MEGA5. Bootstrap values from 100 replicates are indicated at each node. Our PP classes were defined based on the the classification criterion suggested by Singh et al. [13,14].

\section{Gene structure and intron/exon configuration}

To investigate the intron/exon structures of PP genes from maize and rice, we collected useful information from genome annotations of maize and rice from the Maize Genome Sequence Project and TIGR database, respectively. Both DNA sequences and the corresponding coding sequences were loaded into the Gene Structure Display Server (http://gsds.cbi.pku.edu.cn/). For a better visualization and comparison, the $5^{\prime}$ untranslated region (UTR) sequences were removed beforehand.

\section{Chromosomal localization, gene duplication and synteny analysis}

To determine the location of $\mathrm{ZmPP}$ genes on 10 chromosomes, we extracted data concerning gene positions from the Maize Genome Sequence Project. MapDraw 2.2 was used to visualize the locations of $\mathrm{ZmPP}$ genes on maize chromosomes with physical distances in million bp (Mb). We adopted a group-specific color strategy to mark each gene for better visualization and recognition. Tandemly duplicated genes were defined as closely related genes on a single chromosome, with no more than ten intervening genes [2]. In contrast, segmentally duplicated genes were detected by CoGe SynMap program (http://genomevolution.org/CoGe/SynMap.pl). In order to determine the syntenic regions between the rice and maize genomes, the positions of syntenic regions from these two genomes were collected from the results calculated by Synteny Mapping and Analysis Program
(SyMAP 4.0) [24]. The segmental duplications and syntenic regions were finally visualized using Circos 0.62 (http://circos.ca).

The number of nonsynonymous substitutions per nonsynonymous site $(\mathrm{Ka})$ and the number of synonymous substitution per synonymous site (Ks) of duplicated genes were calculated by DnaSP 5.0 [25]. The ratio of non-synonymous to synonymous nucleotide substitutions $(\mathrm{Ka} / \mathrm{Ks})$ between paralogs was analyzed to explore the mechanism of gene divergence after duplication. The dates of the duplication events were calculated by the equation $T=\mathrm{Ks} / 2 \lambda \times 10^{-6}$ Mya, the $\lambda=6.5 \times 10^{-9}$ [26].

\section{Expression analyses by microarray}

To analyze the expression patterns of ZmPPs, the transcriptome data of genome-wide gene expression atlas of maize inbred line B73 were downloaded from the database PLEXdb (http://www.plexdb.org/) with the accession number ZM37. For drought treatments, microarray data were obtained from NCBI Gene Expression Omnibus (GEO) with accession number GSE16567. For each treatment, signal intensity values were normalized and biological replicate samples were averaged to generate $\log 2$ expression values for each gene. Log2-transformed values were loaded into R (15.2) and Bioconductor for expression analysis (http://www.bioconductor.org/). Limma package was applied to data processing, and heatmaps representing log2transformed values were generated with the gplots package [27]. A hierarchical clustering algorithm was applied to determine similar pattern in expression profiles. Microarray data of cold stress were extracted from the ArrayExpress database under the experiment accession number EMTAB-1315. For drought, differentially expressed genes were selected under a very stringent cutoff, with $0.05 P$ value and fold change value of 2 , whereas the response must be at least 2.8-fold in cold stress.

\section{Differential gene expression profiles based on RNA-seq}

To generate the expression profiles of ZmPPs among different organs and development stages, the RNA-seq data were downloaded from the NCBI Short Read Archive with accession number SRP010680 (http://www.ncbi.nlm.nih. gov/sra/). This RNA-seq data analyzed 18 selected tissues representing five organs from maize inbred line B73. Normalized gene expression values were estimated by fragments per kilobase pair of exon model per million fragments mapped (FPKM). Finally, log2-transformed FPKM values from 18 tissues were used to draw heatmaps. To avoid taking the $\log$ of a number less than 1, all such FPKM values were replaced by 1 . Similar to microarray analysis, both limma and gplots packages were used to generate the heatmaps.

RNA-seq data of three types of roots (CR, PR, and SR) under salt stress were fetched from GEO database at 
NCBI under the corresponding accession number GSE53995. Normalized signal intensity values were logtransformed for further analysis. Moreover, 2-fold change was used to test the differentially expressed genes. The up- or down-regulated genes in any tissues were calculated from the average of log-transformed normalized signal values.

\section{Plant materials and stress treatments}

Plants of maize were grown in experimental plots with a 1:1:1 mix of peat:vermiculite:perlite. A growth room with controlled environmental conditions $\left(15 \mathrm{~h}\right.$ light $/ 25^{\circ} \mathrm{C}$, $9 \mathrm{~h} \mathrm{dark} / 20^{\circ} \mathrm{C}$, relative humidity of $55 \%$ ) was used for growing plants. The seedlings were watered daily for about 2 weeks. For drought treatment, 14-day-old seedlings of maize inbred line Ye478 and Han21 were exposed to drought stress for 3 or 4 days, while controls were well watered throughout this period. At the end of treatment period, the volumetric water content of the soil (measured at the depth of approximately $5 \mathrm{~cm}$ from the top of soil level) was approximately $12.5 \%$ for the drought-stressed plants, compared with approximately $30 \%$ for the control plants. For salt treatment, maize inbred line B73 was selected. Seedlings were watered with $200 \mathrm{mM} \mathrm{NaCl}$ for $24 \mathrm{~h}$, after which all stress-treated and control seedlings were harvested, separated into shoots and roots and stored at $-80^{\circ} \mathrm{C}$. Each treatment was replicated three times with 5 to 7 seedlings. For cold treatment, kernels of maize inbred line Huangzao4 and Mol17 were germinated for $3 \mathrm{~d}$ in darkness at $25^{\circ} \mathrm{C}$. Seedlings were transferred to pots containing Knop's solution and were grown in a growth chamber (photoperiod: 14/ $10 \mathrm{~h}$ day/night, light irradiance: $250 \mu$ mol quanta $\mathrm{m}^{-2} \mathrm{~s}^{-1}$, temperature: $24 / 25^{\circ} \mathrm{C}$ and relative humidity $60 / 80 \%$ ). When the third leaf was fully developed, half of the plants were transferred to $8 / 6^{\circ} \mathrm{C}$ (day/night) without changing the other conditions; the other half were grown in the same conditions as before (control plants).

Seeds of Arabidopsis thaliana were sterilized with 2\% bleach for $20 \mathrm{~min}$, plated on Murashige and Skoog medium (MS; Sigma-Aldrich), chilled at $4^{\circ} \mathrm{C}$ for $3 \mathrm{~d}$, and transferred to a growth room at 19 to $23^{\circ} \mathrm{C}$ with a $16 \mathrm{~h} /$ $8 \mathrm{~h} \mathrm{light/dark} \mathrm{photoperiod.}$

\section{RNA extraction and real-time PCR}

To validate the RNA-seq data for selected genes under abiotic stresses, real-time PCR was carried out. The leaves and roots of the maize seedlings were gathered, immediately frozen in liquid nitrogen and then stored at a temperature of $-80^{\circ} \mathrm{C}$. Subsequently, total RNA was isolated from selected tissues using Trizol reagent, and the use of DNase I treatment to remove any genomic DNA contamination from RNA samples. About $2 \mu \mathrm{g}$ of total RNA was reverse transcribed using a Takara RNA
Kit to generate the single-stranded cDNA. Real-time PCR was performed on iCycler iQ5 Multicolor real-time PCR detection system (Bio-Rad) by using the Power SYBR Green PCR Master Mix (APPlied Biosystems). Two biological replicates of each sample were used and three technical replicates were performed for each biological replicate. The thermal cycling conditions are as follows: $50^{\circ} \mathrm{C}$ for $2 \mathrm{~min}, 95^{\circ} \mathrm{C}$ for $10 \mathrm{~min}, 40$ cycles of $95^{\circ} \mathrm{C}$ for $15 \mathrm{~s}$, and $60^{\circ} \mathrm{C}$ for $1 \mathrm{~min}$. The relative quantification method $2^{-\Delta \mathrm{Ct}}$ was used to evaluate quantitative variation between replicates. For drought and cold stresses, maize ubiquilin-1 (UBQ1) gene was used as internal control, while ACTIN7 was an internal control for salt stress.

For fptp1 mutant analysis, total RNA was extracted from rosette leaves of 40 day-old plants. ACTIN8 was used as an internal control. The real-time PCR experiment was repeated for three independent biological replicates. Finally, the ABi7500 real-time PCR system with the RealMasterMix (SYBR Green I) (Takara) was used for performing real-time PCR. Primer pairs used in this study were listed in Additional file 1. The fptp1 mutant was obtained from Institute National de la Recherche Agronomique (INRA).

\section{Over-expression of FPTP1 in Arabidopsis and analysis of ABA synthetase gene expression}

To generate Arabidopsis FPTP1 over-expression transgenic plants, the coding sequence of FPTP1 were amplified and inserted into the modified pCAMBIA1300-Super vector under control of $35 \mathrm{~S}$ promoter. These vectors were transformed into Agrobacterium strain GV3101 and subsequently introduced into the Arabidopsis wile-type plants.

With the aim of identifying the contribution of PTPs in ABA accumulation, RNA was extracted using an RNeasy Plant Minikit (QIAGEN), and cDNA was synthesized using M-MLV Reverse Transcriptase (M1701) (Promega). $1 \mu \mathrm{g}$ RNA was mixed thoroughly with $1 \mu \mathrm{g}$ Oligo $(\mathrm{dT})_{15}$, and treated at $70^{\circ} \mathrm{C}$ for $5 \mathrm{~min}$. Subsequently, the following were added: $5 \mu \mathrm{L}$ M-MLV buffer, $1.25 \mu \mathrm{L}$ dNTP Mix, $1 \mu \mathrm{L}$ M-MLV, and $0.6 \mu \mathrm{L}$ RNase inhibitor. The resulting $25 \mu \mathrm{L}$ reaction was incubated for $1 \mathrm{~h}$ at $37^{\circ} \mathrm{C}$. PCR primers were showed in Additional file 1. PCR cycles included $94^{\circ} \mathrm{C} 5 \mathrm{~min},\left(94^{\circ} \mathrm{C} 45 \mathrm{~s}, 58^{\circ} \mathrm{C} 45 \mathrm{~s}, 72^{\circ} \mathrm{C} 1 \mathrm{~min}\right) 35$ cycles, $72^{\circ} \mathrm{C} 7 \mathrm{~min}$.

\section{Subcellular location of ZmPP1 and estrogen treatment of ZmPP1}

For subcellular localization of $Z m P P 1$, its cDNA sequence was cloned into pEZS-NL transient expression vector to generate $\mathrm{pEZS-NL-ZmPP1}$. Particle bombardmentmediated transient expression of ZmPP1-GFP fusion protein in onion epidermal cells were investigated by laser scanning confocal microscopy. As for GUS staining, the cDNA of ZmPP1 was cloned into pSuper-1391 vector and 
then introduced into the GV3101. Floral dip method can efficiently generate the Arabidopsis transgenic plants. Then the transgenic plants were screened on solid plates containing $50 \mathrm{mg} / \mathrm{mL}$ hygromycin.

We have developed an estrogen receptor-based chemicalinducible system for ZmPP1 transgenic Arabidopsis. The amplified ZmPP1 cDNA sequence was cloned into pER8, of which the pER8 vector was provided by N.-H.Chua and B. Ulker [28]. Transformations of Arabidopsis were performed by the floral dip method using GV3101. Overexpression of $Z m P P 1$ in the transgenic lines was induced by spraying the seedling with 5 or $10 \mu \mathrm{M} \beta$-estradiol.

\section{Promoter activity and computational prediction of miRNA protein-coding target transcripts}

To find out putative cis-acting elements in the promoters of putative $\mathrm{ZmPP}$ genes, the 1,000 bp upstream promoter regions of all ZmPPs were used to search for known stress-responsive cis-elements by PlantCARE database (http://bioinformatics.psb.ugent.be/webtools/plantcare/html/). Target-align [29], a miRNA target prediction tool, was used to predict putative miRNA target genes. For the maize protein-coding transcripts, the predicted cDNA of the longest consensus maize transcript were used. A total of 321 mature miRNA sequences downloaded from miRBase database (release 20) [30] were reverse complemented and matched against the indexed maize transcript database. With the default setting, a score cut-off of $\geq 75$ and mismatch $\leq 4$ nucleotides were applied in the prediction.

\section{Results and discussion}

\section{Identification of PPs in maize genome}

Previously, 112 and 132 PP genes were identified from Arabidopsis and rice, respectively $[13,14]$. To explore the occurrence and size of the PP family in maize, HMMER program was used to search against the maize proteomics database. Further, the presence of catalytic domain was confirmed by SMART and Pfam databases. From maize genome, we identified a total of 159 putative PPencoding genes following the removal of those sequences with an incomplete catalytic domain, suggesting that the size of maize PP family was larger when compared with that in Arabidopsis and rice. For simplicity, the $159 \mathrm{ZmPP}$ genes were renamed from $Z m P P 1$ to $Z m P P 159$ according to their exact positions on maize chromosomes 1-10 and from top to bottom. The identified PP genes in maize encode proteins ranging from 131 to 1264 amino acids (aa). With the WoLF PSORT analysis, most PPs were predicted to locate in nucleus and chloroplast as well as other organelles. ExPaSy analysis suggested that the $\mathrm{ZmPP}$ proteins had large variations in isoelectric point $(\mathrm{pI})$ values (ranging from 4.46 to 9.8) and molecular weights (ranging from 14,245.15 to $90,157.14 \mathrm{Da}$ ). All of the related information on ZmPPs is listed in the Additional file 2.

\section{Phylogenetic analysis and classification of the ZmPPs}

The catalytic domains of PPs are diverse even if the catalytic cores of all eukaryotic PKs share extensive similarities in both primary and three-dimensional structures. With the aim of analyzing the phylogenetic relationships of $159 \mathrm{ZmPPs}$ among maize, phylogenetic tree was carried out based on the catalytic domains by NJ algorithm (Figure 1). Based on the sequence similarities of the $\mathrm{ZmPP}$ gene sequences and the classification of PP genes from Arabidopsis and rice, the phylogenetic tree divided the maize PP family proteins into three major classes, namely, PP2C, PTP and PPP (PP2A). PP2C was evolutionarily related to the major class of Ser/Thr PPs with 104 genes, whereas PTP and PP2A comprised of 29 and 26 members, respectively. Interestingly, all the members of $\mathrm{PP} 2 \mathrm{C}$ class formed a single major group and this group could be further classified into 11 subgroups except some ambiguous branches. Each subgroup represents a subfamily of PP2C and is designated from A-K according to Xue et al. [31] and Singh et al. [14]. Group J PP2C did not include any rice and maize PPs but contained only members from Arabidopsis, suggesting that they might have been lost both in rice and maize after being originated from common ancestor.

In terms of the previous studies [1,31], plant lacks typical tyrosine kinase, and most plant PPs (67\%) have Ser/ Thr specificity. It seems that plants prefer to use Ser/Thr as substrates for PKs and PPs, in sharp contrast with the case in human [32]. Three genes belonging to LMWP (ZmPP14), PTPL (ZmPP47) and CDC25 (ZmPP145) classes were positioned separately. PP2As, which were ubiquitous enzymes in eukaryotes, formed another single major class. Phylogenetic tree obtained from Arabidopsis, rice and maize showed very similar topologies and subfamily organization with individual maize tree (Additional file 3). It is noteworthy that the number of maize PP genes was generally overrepresented than that of Arabidopsis and rice in almost all classes.

\section{Gene structure analysis and intron/exon arrangement}

As a evolutionary relic, it is noteworthy that intron/exon arrangement carries the imprint of the evolution of a gene family, and may provide insight into their evolutionary mechanisms underlying the origin of gene families [33]. With the purpose of understanding the structure diversity and evolution of ZmPP gene family, we analyzed the intron/exon structure, intron position and phase. A detail illustration of the intron/exon structure was shown in Additional file 4. It was found that the great majority of introns were positioned in the coding sequences of $\mathrm{ZmPP}$ genes. Notably, in PP2C class, the intron/exon structures 


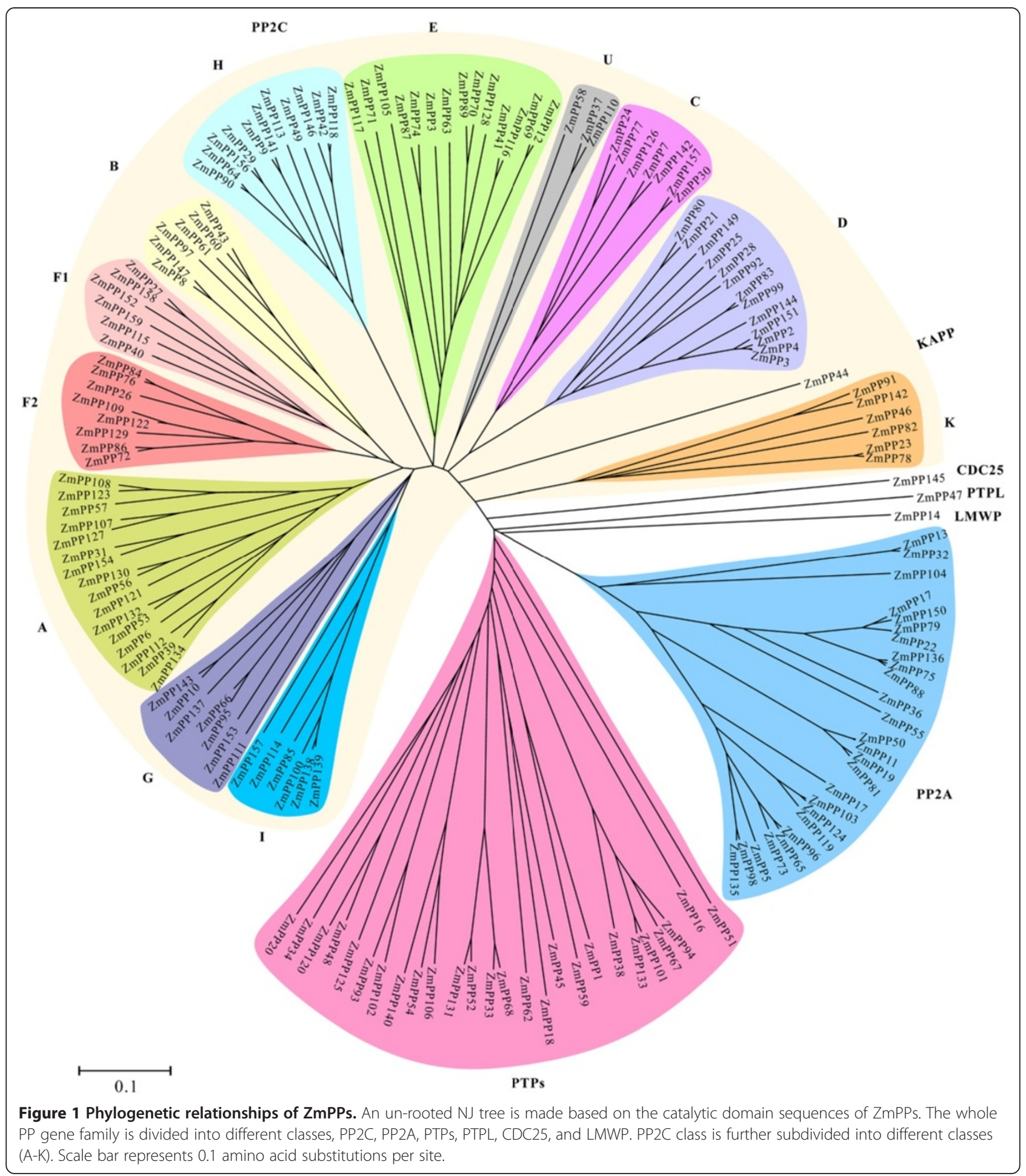

were different between subclasses and correlated with the architecture of protein sequence and phylogenetic analysis. However, it was notable that a similar gene structure was found in each group, while 4 members of the 104 ZmPP2C genes (ZmPP39, -112, -91, and -142) had no introns. This indicated that the intron patterns, which correlate well with the phylogentic clades, strongly support their close evolutionary relationships among the ZmPP2C genes within the same subclass. Among those having introns, the number of introns within the open reading frame (ORF) ranged from 1 to 12, showing a great difference in the $\mathrm{ZmPP} 2 \mathrm{C}$ class. A great degree of variation in 
the number of introns exists in groups E, G and K, while the number of introns in the rest groups changes within a small range, mostly from 1 to 3 . In rice, similar results of gene structure analysis were given in Additional file 5, indicating that similar intron loss or gain events occurred during expansion and structural evolution of 2C-type protein phosphatase may exist in monocotyledon.

In PTPs, introns are not equally distributed across family. Additionally, we found that nearly all members (expect ZmPP73) have introns, while the number of introns varies widely within PP2A group. We further analyzed the exon/intron configuration of the paralogous pairs in PP gene family to obtain traceable intron gain or loss information (Additional file 5). Despite the structural conservation of intron/exon arrangement found in some paralogous pairs, others exhibited extensive variation. What's more, growing evidence has showed a functional link between the structural diversity of gene members and the evolution of multiple gene families, while intron loss or gain can be an important step in generating structural diversity and complexity [34].

\section{Chromosomal location and gene duplication of ZmPP genes}

To determine the genomic distribution of the $\mathrm{ZmPP}$ genes and study their evolution in the context of whole genome duplication, we mapped each gene on maize chromosomes based on their corresponding coordinates. As shown in Figure 2, ZmPPs are almost unevenly distributed across the ten chromosomes. All members of class PP2C are distributed across 10 maize chromosomes. In contrast, no PTPs and PP2As were found to be located on chromosome 7 and 10, respectively (Figure 2).

Gene duplication is prominent in eukaryotic evolution, because duplicated genes provide the raw materials for evolving a new gene, which in turn facilitate the generation of novel gene functions. Compared with most other eukaryotes, plants have substantially higher gene duplication rates in the adaptive response to environmental stimuli [35]. Previous study revealed that maize genome underwent a whole-genome duplication event (WGD) that resulted in the presence of two sub-genomes and $~ 30 \%$ of genes retained copies in both sub-genomes [36]. The duplication mechanism including transposition, segmental duplication, replicative transposition, or even WGD underlying gene family expansion are similar between eukaryotes. To clarify the probable relationship between $\mathrm{ZmPP}$ genes and potential gene duplication within the genome, we analyzed the occurrence of tandem duplication and large-scale segmental duplication during the evolution. In all, 15 ( 9.4\%) ZmPP genes were found to be tandem repeats with a maximum number of ten intervening spacer genes (Table 1). These $15 \mathrm{ZmPP}$ genes were represented in 9 distinct tandem duplicated gene clusters, with one cluster containing 3 tandem genes and the rest of clusters possessing 2 tandem genes. It is notable that genes in one duplicated cluster may come from different group. For instance, ZmPP144 was grouped into PP2C, but $Z m P P 145$ was a member of PTPs. Hence, it is speculated that tandem duplications have an indispensable role in the evolution of maize PP gene family. Additionally, previous study showed that most plants were diploidized polyploids and retained numerous duplicated chromosomal blocks in their genomes, segmental duplication occurred most frequently in plants [37]. To evaluate the contribution of large segmental duplications in the expansion of ZmPP-encoding gene family, CoGe SynMap program was used to detect segmental duplicated genes. We noted that 38 segmental duplication events in maize were observed, which were originated from a polyploidy event occurred around 15 to 20 million years ago (Table 1 ). Interestingly, all genes in segmental duplicated pairs were from the same classes. Thus, we speculated that segmental duplications exclusively contributed to the expansion of PP gene family in the maize genome. Taken together, our analyses suggested that large-scale segmental duplication appear to have prominently contributed to the current complexity of the maize PP family. It is consistent with the duplicative mechanism of PP gene family in rice [14].

To illuminate the divergence after gene duplication, the ratios of $\mathrm{Ka} / \mathrm{Ks}$ were estimated for all 48 duplicated pairs. Usually, $\mathrm{Ka} / \mathrm{Ks}>1$ means positive selection; $\mathrm{Ka} / \mathrm{Ks}=1$ means neutral evolution; while $\mathrm{Ka} / \mathrm{Ks}<1$ means purifying selection $[38,39]$. $\mathrm{Ka} / \mathrm{Ks}$ ratios of most duplicated pairs, regardless of whether they are orthologs and paralogs, were less than 1 , subjecting to purifying selection. Nevertheless, there are 5 segmentally duplicated pairs suffered positive selection with $\mathrm{Ka} / \mathrm{Ks}$ ratios greater than 1 . Among these duplicated pairs, the average $\mathrm{Ka} / \mathrm{Ks}$ value of segmental duplication was very similar to those in tandem duplication $(\sim 0.4)$, indicating that most duplicated $\mathrm{ZmPP}$ genes are under strong purifying selection pressure. The duplication events for the segmental duplicated genes were estimated to occur approximately 24. Mya, while that in tandem duplicated pairs were estimated to occur approximately 37 Mya, indicating that the segmental duplication events might occur before the emergence of tandem duplication events. Therefore, the segmental and tandem duplication events played essential roles in evolutionary expansion of PP family in maize.

\section{Expression profiles of maize PP genes and their potential functions in different tissues and developmental stages}

Recent advances in functional analyses of PPs have revealed the importance of some PP genes in the life cycles of both Arabidopsis and rice [14,40], however, their roles in maize remained unclear. Aim for achieving gene expression patterns of $Z m P P s$ in diverse growth phases, we 


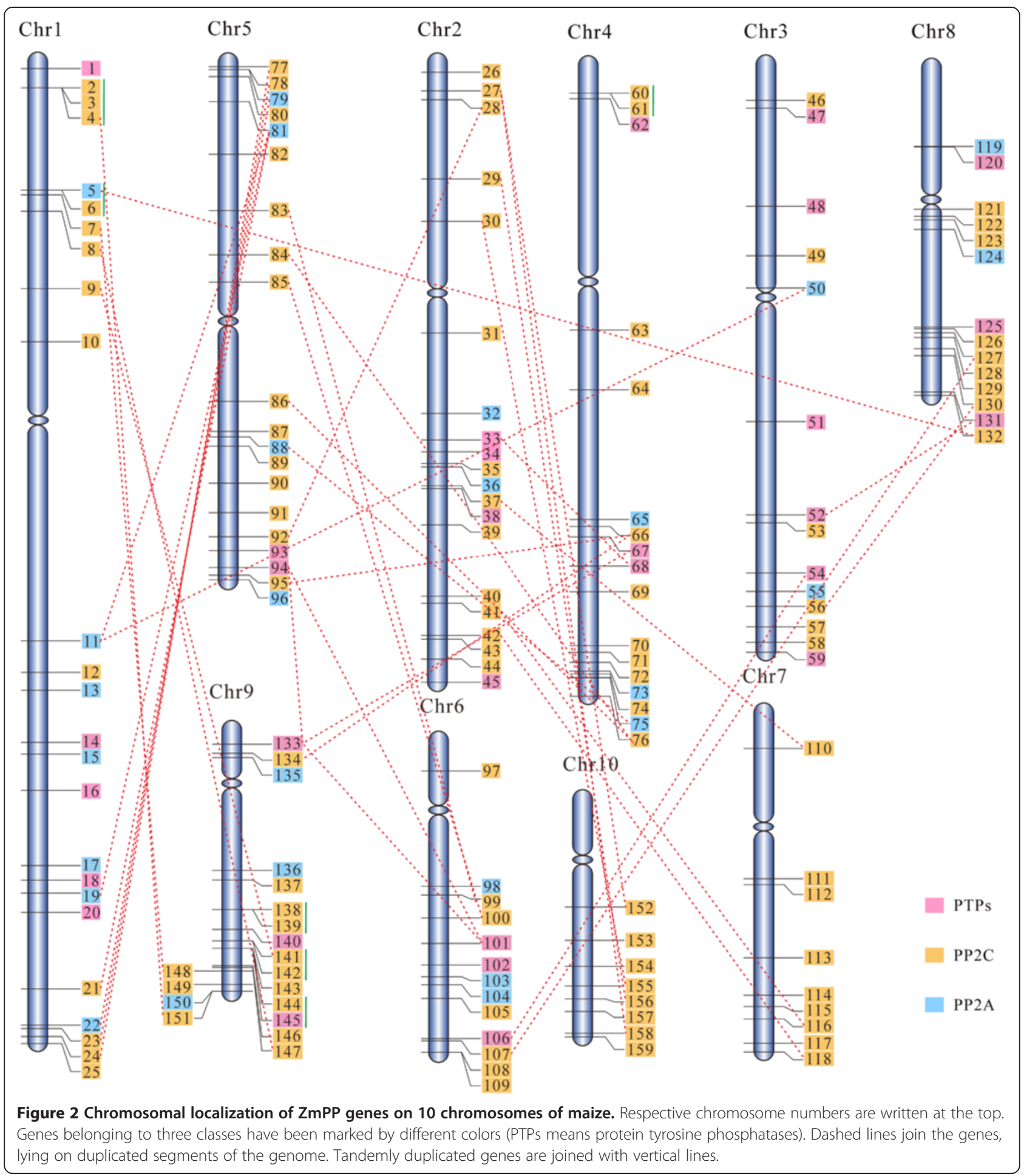

downloaded the genome-wide expression data of maize inbred B73 from PLEXdb and analyzed the expression profiles of $Z m P P s$ across the 60 different developmental stages of 11 organs. Following whole-chip data processing, the $\log 2$ values of $154 \mathrm{PP}$ genes were showed as heatmap (Additional file 6). From Additional file 6, we found that $Z m P P s$ were implicated in a wide range of plant development processes, including root development, leaf development gradient and flower induction. In addition to conducting microarray analysis, RNA-seq data with 18 tissues representing distinct stages of maize plant development were selected for analysis. A total of 
Table $1 \mathrm{The} \mathrm{Ka} / \mathrm{Ks}$ ratios and estimated absolute dates for the duplication events between the duplicated ZmPP genes

\begin{tabular}{|c|c|c|c|c|c|}
\hline Duplicated pair & $\mathrm{Ka} / \mathrm{Ks}$ & Date (Mya) & Duplicate type & Purifying selection & Group \\
\hline ZmPP53/ZmPP132 & 0.2091 & 13.02307692 & Segmental & Yes & PP2C/PP2C \\
\hline ZmPP56/ZmPP130 & 0.35128 & 13.86153846 & Segmental & Yes & $\mathrm{PP} 2 \mathrm{C} / \mathrm{PP} 2 \mathrm{C}$ \\
\hline ZmPP107/ZmPP127 & 0.26416 & 18.2 & Segmental & Yes & PP2C/PP2C \\
\hline ZmPP134/ZmPP66 & 0.10751 & 86.57692308 & Segmental & Yes & $\mathrm{PP} 2 \mathrm{C} / \mathrm{PP} 2 \mathrm{C}$ \\
\hline ZmPP8/ZmPP147 & 0.13034 & 20.53846154 & Segmental & Yes & $\mathrm{PP} 2 \mathrm{C} / \mathrm{PP} 2 \mathrm{C}$ \\
\hline ZmPP7/ZmPP148 & 0.39908 & 10.06153846 & Segmental & Yes & PP2C/PP2C \\
\hline ZmPP24/ZmPP77 & 1.29469 & 5.507692308 & Segmental & No & PP2C/PP2C \\
\hline ZmPP30/ZmPP155 & 0.15067 & 12.1 & Segmental & Yes & PP2C/PP2C \\
\hline ZmPP4/ZmPP151 & 0.10791 & 16.53846154 & Segmental & Yes & $\mathrm{PP} 2 \mathrm{C} / \mathrm{PP} 2 \mathrm{C}$ \\
\hline ZmPP21/ZmPP80 & 0.14157 & 21.40769231 & Segmental & Yes & PP2C/PP2C \\
\hline ZmPP28/ZmPP92 & 0.16562 & 67.48461538 & Segmental & Yes & $\mathrm{PP} 2 \mathrm{C} / \mathrm{PP} 2 \mathrm{C}$ \\
\hline ZmPP83/ZmPP99 & 0.08266 & 14.23846154 & Segmental & Yes & PP2C/PP2C \\
\hline ZmPP41/ZmPP116 & 0.11765 & 16.73846154 & Segmental & Yes & PP2C/PP2C \\
\hline ZmPP27/ZmPP152 & 1.60306 & 16.60769231 & Segmental & No & PP2C/PP2C \\
\hline ZmPP152/ZmPP158 & 2.32622 & 11.17692308 & Segmental & No & $\mathrm{PP} 2 \mathrm{C} / \mathrm{PP} 2 \mathrm{C}$ \\
\hline ZmPP158/ZmPP27 & 0.21536 & 12.32307692 & Segmental & Yes & PP2C/PP2C \\
\hline ZmPP72/ZmPP86 & 0.11398 & 16.06153846 & Segmental & Yes & PP2C/PP2C \\
\hline ZmPP76/ZmPP84 & 0.09925 & 15.34615385 & Segmental & Yes & PP2C/PP2C \\
\hline ZmPP122/ZmPP129 & 0.19247 & 65.58461538 & Segmental & Yes & PP2C/PP2C \\
\hline ZmPP95/ZmPP66 & 0.07717 & 30.7 & Segmental & Yes & $\mathrm{PP} 2 \mathrm{C} / \mathrm{PP} 2 \mathrm{C}$ \\
\hline ZmPP9/ZmPP141 & 0.18169 & 11.17692308 & Segmental & Yes & PP2C/PP2C \\
\hline ZmPP29/ZmPP156 & 0.16686 & 13.23076923 & Segmental & Yes & $\mathrm{PP} 2 \mathrm{C} / \mathrm{PP} 2 \mathrm{C}$ \\
\hline ZmPP42/ZmPP118 & 0.10881 & 14.13846154 & Segmental & Yes & PP2C/PP2C \\
\hline ZmPP85/ZmPP100 & 1.28064 & 17.76153846 & Segmental & No & $\mathrm{PP} 2 \mathrm{C} / \mathrm{PP} 2 \mathrm{C}$ \\
\hline ZmPP23/ZmPP78 & 0.17848 & 23.23076923 & Segmental & Yes & PP2C/PP2C \\
\hline ZmPP37/ZmPP110 & 0.25391 & 15.23846154 & Segmental & Yes & PP2C/PP2C \\
\hline ZmPP33/ZmPP68 & 0.18219 & 16.76153846 & Segmental & Yes & DSP/DSP \\
\hline ZmPP52/ZmPP131 & 0.12905 & 14.00769231 & Segmental & Yes & DSP/DSP \\
\hline ZmPP54/ZmPP106 & 0.24256 & 70.78461538 & Segmental & Yes & DSP/DSP \\
\hline ZmPP67/ZmPP133 & 0.33671 & 44.04615385 & Segmental & Yes & DSP/DSP \\
\hline ZmPP94/ZmPP133 & 2.1275 & 11.94615385 & Segmental & No & DSP/DSP \\
\hline ZmPP94/ZmPP101 & 1.8272 & 13.8 & Segmental & No & DSP/DSP \\
\hline ZmPP101/ZmPP133 & 0.13011 & 13.36153846 & Segmental & Yes & DSP/DSP \\
\hline ZmPP11/ZmPP50 & 0.05879 & 15.17692308 & Segmental & Yes & PP2A/PP2A \\
\hline ZmPP11/ZmPP81 & 0.01588 & 105.6230769 & Segmental & Yes & $\mathrm{PP} 2 \mathrm{~A} / \mathrm{PP} 2 \mathrm{~A}$ \\
\hline ZmPP19/ZmPP81 & 0.05683 & 14.75384615 & Segmental & Yes & PP2A/PP2A \\
\hline ZmPP22/ZmPP79 & 0.00792 & 13.59230769 & Segmental & Yes & PP2A/PP2A \\
\hline ZmPP75/ZmPP88 & 0.05525 & 9.884615385 & Segmental & Yes & PP2A/PP2A \\
\hline ZmPP2/ZmPP3 & 0.10884 & 8.269230769 & Tandem & Yes & PP2C/PP2C \\
\hline $\mathrm{ZmPP} 2 / \mathrm{ZmPP} 4$ & 0.12221 & 5.853846154 & Tandem & Yes & PP2C/PP2C \\
\hline ZmPP3/ZmPP4 & 0.1199 & 6.030769231 & Tandem & Yes & PP2C/PP2C \\
\hline ZmPP5/ZmPP6 & 0.96739 & 84.93076923 & Tandem & Yes & PP2A/PP2C \\
\hline ZmPP60/ZmPP61 & 0.36275 & 41.66923077 & Tandem & Yes & $\mathrm{PP} 2 \mathrm{C} / \mathrm{PP} 2 \mathrm{C}$ \\
\hline ZmPP119/ZmPP120 & 0.77549 & 49.03076923 & Tandem & Yes & PP2A/DSP \\
\hline
\end{tabular}


Table $1 \mathrm{The} \mathrm{Ka} / \mathrm{Ks}$ ratios and estimated absolute dates for the duplication events between the duplicated ZmPP genes (Continued)

\begin{tabular}{llllll}
\hline ZmPP138/ZmPP139 & 0.43648 & 2.361538462 & Tandem & Yes & PP2C/PP2C \\
ZmPP141/ZmPP142 & 0.57337 & 85.86153846 & Tandem & Yes & PP2C/PP2C \\
ZmPP144/ZmPP145 & 0.68466 & 50.03076923 & Tandem & Yes & PP2C/DSP \\
\hline
\end{tabular}

${ }^{*} \mathrm{Ka} / \mathrm{Ks}<1$ means purifying selection.

$152 \mathrm{ZmPPs}$ were reliably detected, while the remaining 7 members were undetected (Figure 3A, Additional file 7). We also calculated the $\mathrm{CV}$ value $\left(\mathrm{CV}=\mathrm{S} / \mathrm{X}_{\text {mean }}\right.$, where $\mathrm{S}$ represents the standard deviation and $\mathrm{X}_{\text {mean }}$ indicates the mean expression of a gene across all the tissues) of each gene to elucidate the expression profiles of differentially expressed genes [41]. Based on their expression levels and CV values, these detected PP genes were categorized into two lineages. Lineage I showed higher expression levels, but lower $\mathrm{CV}$ values compared with lineage II. As shown in Additional file 7, genes with higher $\mathrm{CV}$ values displayed higher fluctuation in their expression levels.

Recently, a systematic survey of morphology and histology of young seedling root in maize hybrids and their parental inbred lines has been performed [42]. Before this, most of our knowledge about the complexity of auxin signaling in regard to root development was based on the molecular and physiological analyses in Arabidopsis, which laid the groundwork for the discovery of parallel pathways using maize as a model for monocot plants. To better understand the role of auxin in maize root development, we searched for some key factors in the signaling pathways (Additional file 8). In comparison with Arabidopsis and rice, less development-regulated genes and their functions in maize have been characterized in detail. In our study, identification of key genes involved in root development signaling pathway in maize, such as auxin transporters (PIN) and auxin response factors (ARF) were performed using HMM-based searches. All genes involved in this developmental signaling pathway were showed in Additional file 9. It has been reported that PP2As are required for asymmetric auxin distribution in seedling roots of Arabidopsis [43]. During male and female inflorescence differentiation and kernel development, the transcripts of ZmPIN gene were showed to have overlapping expression domain in the root apex [44]. Moreover, the inhibition of PP2A leads to a preferential apical PIN targeting [43]. In our microarray data, ZmPP65 was relatively high expressed in root, indicating that ZmPP65 might play an important role in auxin-induced root development. Interestingly, another two genes (ZmPP67 and ZmPP94), which belong to PP2C class, exhibited high expression levels in root. Thus, we hypothesize that except for PP2A, other classes of PP genes may participate in root development as well, such as PP2C. Moreover, expression patterns of other PP genes involved in maize root developmental pathway were analyzed as well (Figure 3A, Additional file 9). Most of them were highly expressed in root, which were in accordance with the results that other PP genes except PP2As play roles in root development as well.

It has been reported that maize leaves are characterized by clear proximal/distal domains as seen in the morphological differences of the sheath and blade. In our research, it was noteworthy that two genes (ZmPP145 and ZmPP58), belonging to lineage I, were found to display high expression levels at V5_Tip of stage-2 Leaf, while ZmPP58 was highly expressed at V9_Eighth leaf. It is speculated that ZmPP145 and ZmPP58 may play critical roles in early leaf developmental stages (V5 and V9). To provide a broader insight into the maize leaf development, four representative zones of the leaf blade were selected for deeper transcriptomic analysis (Additional file 10) [45]. Of $159 \mathrm{ZmPP}$ genes, a total of 88 genes were expressed at all four sampled sections of the maize leaf. Most of them were upregulated in all four regions of maize leaf. Especially, ZmPP47 showed increased transcript abundance in the basal (base, $1 \mathrm{~cm}$ above the leaf three ligule) and transitional zones $(-1 \mathrm{~cm}, 1 \mathrm{~cm}$ below the leaf two ligule), while $Z m P P 58$ and ZmPP133 were highly expressed in the maturing $(+4 \mathrm{~cm}, 4 \mathrm{~cm}$ above the leaf two ligule) and mature zones (tip, $+1 \mathrm{~cm}$ below the leaf three tip), which might participate in the transcriptional regulation of carbon fixation pathways. Arabidopsis PLL4 (a member of PP2C class) was reported to play a part in the regulation of leaf development, while its homolog in maize ( $Z m P P 24)$ showed higher expression in the maturing and mature zones as well. Strikingly, 4 genes (ZmPP48, -113, -120 and -146) were obviously down-regulated in the maturing and mature zones with their functions in leaf development remain unknown. Overall, genes with specific expression patterns in this study might play critical roles in leaf development.

Morphogenesis is referred to as a process in developmental biology in which organisms develop their characteristic shapes. Furthermore, flowering and architecture development are two key processes in plant morphogenesis, which are of particular significances in crop. Modulation of the life cycle by photoperiodic signaling is a fundamental mechanism for plant to cope with the seasonal changes. As reported by Dong et al. [46], both environmental and endogenous factors control the shoot 


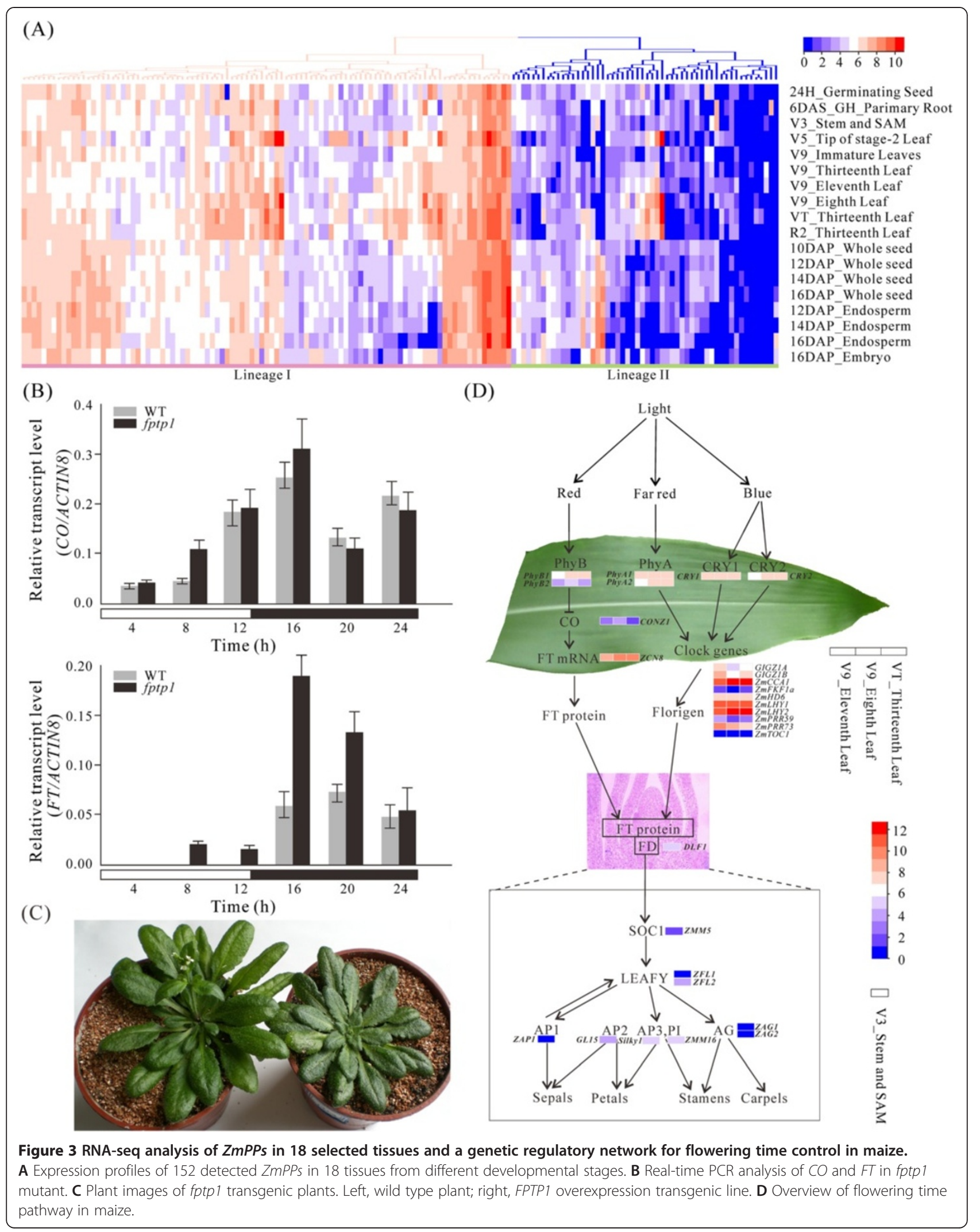


apical meristem (SAM) transition from the vegetative to the reproductive stage. In Figure 3A, ZmPP67 was expressed at a relative high level in both root and SAM, suggesting that ZmPP67 might participate in floral transition. To validate the exact role of $Z m P P 67$ in maize flowering time pathway, the function of Arabidopsis FLOWERING ASSOCIATED PTPase1 (FPTP1, Genebank accession number: FJ605097), which is homologous to $Z m P P 67$, was analyzed by mutant analysis. $C O$, a circadian-regulated gene, and FLOWERING LOCUS T $(F T)$, belonging to the $\mathrm{BBX}$ and PEBP family, respectively, contribute to the photoperiod flowering control in plant [47]. As showed in Figure 3B, mutation in FPTP1 significantly promoted $F T$ expression but had no obvious effect on $C O$ expression. Furthermore, over-expression of FPTP1 caused delayed flowering (Figure $3 C$ ). Thus, we suspected that ZmPP67 may function in the upstream of FT.

It was reported that many of the flowering time pathways in Arabidopsis and rice are conserved in maize [47]. Through a genetic and molecular model for flower development in Arabidopsis, a possible flowering time pathway in maize was proposed (Figure 3D). Light is the most important environmental cue implicated in the regulation of flowering time in plants. In maize, a reduced light response is associated with the development of early flowering inbred lines [48]. Phytochromes and cryptochromes are the primary red/far-red and blue light photoreceptors, respectively, with three pairs in our study, PhyB1/2, PhyA1/2, and CRY1/2. In our expression profiles of genes involved in flower induction (Figure 3D), PhyA, PhyB and $C R Y 1 / 2$ were expressed slightly higher levels in leaves compared with other tissues. Particularly, expressions of PhyB1 and PhyB2 are highly similar to that of PhyA1 and PhyA2 genes. Previous study showed that $P h y B$ regulates CO protein, but not mRNA level [49]. $C O$ coordinates light and clock input in leaves to trigger the expression of florigen gene FT. Consequently, we suspected that the effect of $C O$ on $F T$ expression must result from a posttranscriptional regulation. DLF1, homologous to Arabidopsis FD, form a complex with ZCN8 (maize FT protein) to activate downstream floral organ identity genes, such as $Z M M 5$, the homolog of Arabidopsis SOC1 in maize. Then, $A P 1 / 3, P I$ and $A G$ were the downstream of $L E A F Y$ in Arabidopsis, and the homologs in maize were identified in this study as well as their expression patterns were analyzed. It was showed that DLF1, ZMM5, ZFL2 (LEAFY), GL15 (AP2), Silky1 (AP3), and ZMM16 (PI) were relatively highly expressed in SAM compared with other tissues, while other genes in the downstream of LEAFY, including ZAP1 (AP1) and ZAG1/2 (AG) had lower expression. All genes involved in the flowering time signaling pathway were showed in Additional file 11.

In addition to RNA-seq analysis in 18 representative tissues during maize development, another RNA-seq study of 12 maize reproductive tissues that represent male, female and developing seed was carried out. Overall, $96 \%$ (153) of ZmPPs were detected in reproductive transcriptome (Additional file 12). It was noteworthy that $Z m P P 37,-76,-81,-84,-90$, and -110 were significantly highly expressed in pollen, notably, ZmPP76 and $Z m P P 84$ were specially expressed in anther. Additionally, $Z m P P 15,-63$ and -97 had relatively high expression values in both anther and pollen. Hence, genes with expression restricted to specific tissues within this study are strong candidates for further analysis to unravel the mechanisms of vegetative and reproductive growth.

\section{Expression profiles of ZmPPs under abiotic stresses Expression analysis of ZmPP genes under salt stress}

Plants encounter a wide range of environmental insults, which pose serious threat to crop production. Among all the abiotic stresses, salinity represents the major constraint for agricultural productivity. Maize is a salt-sensitive crop plant and responds to salt stress via complex changes at both the transcriptional and post-transcriptional levels. Plant roots are highly sensitive organ and induce primary response to salt stress. To better understand the different responding mechanisms to salt stress in maize root, examination of three types of roots (CR, PR, and SR) under salt treatment was performed. As shown in the Figure 4A, three specific genes were significantly and differentially expressed under salt stress, namely, ZmPP149, ZmPP66, and $Z m P P 127$. Surprisingly, all of them are the members of PP2C class, and ZmPP66 is a member of Group G PP2C. It has been demonstrated that AtPP2CG1 (Arabidopsis thaliana protein phosphatase $2 \mathrm{C}$ G Group 1) can positively regulate salt-tolerance of Arabidopsis in ABA-dependent manner [10]. Intriguingly, ZmPP10, which is homologues to AtPP2CG1, showed significantly upregulated expression in $\mathrm{PR}$. In addition to these three specially expressed genes (ZmPP149, ZmPP66, and ZmPP127), ZmPP107, a member of Group A PP2C, was significantly up-regulated in $\mathrm{CR}$ and SR, indicating that Group A PP2C may play a part in salt stress except for its role in drought stress condition.

To clearly understand the roles of ZmPPs in salt stressinduced signaling pathway, salt stress signaling and mechanisms of maize salt tolerance were analyzed. Salt stress involves both osmotic stress and ionic stress that limit the efficiency of crops and especially quantity and quality of their metabolic (secondary plant products) products. Osmotic stress can rapidly increase ABA biosynthesis, and regulate ABA-dependent stress response pathway [50]. The expression patterns of key genes involved in ABA biosynthesis showed that VP14 (ZmNCED1) and ZmAO2 (the homolog of Arabidopsis $A A O 3$ ) were significantly upregulated in $\mathrm{CR}$ and SR (Figure $4 \mathrm{~B}$ ). Several salt stressinducible genes were identified in this study and showed 


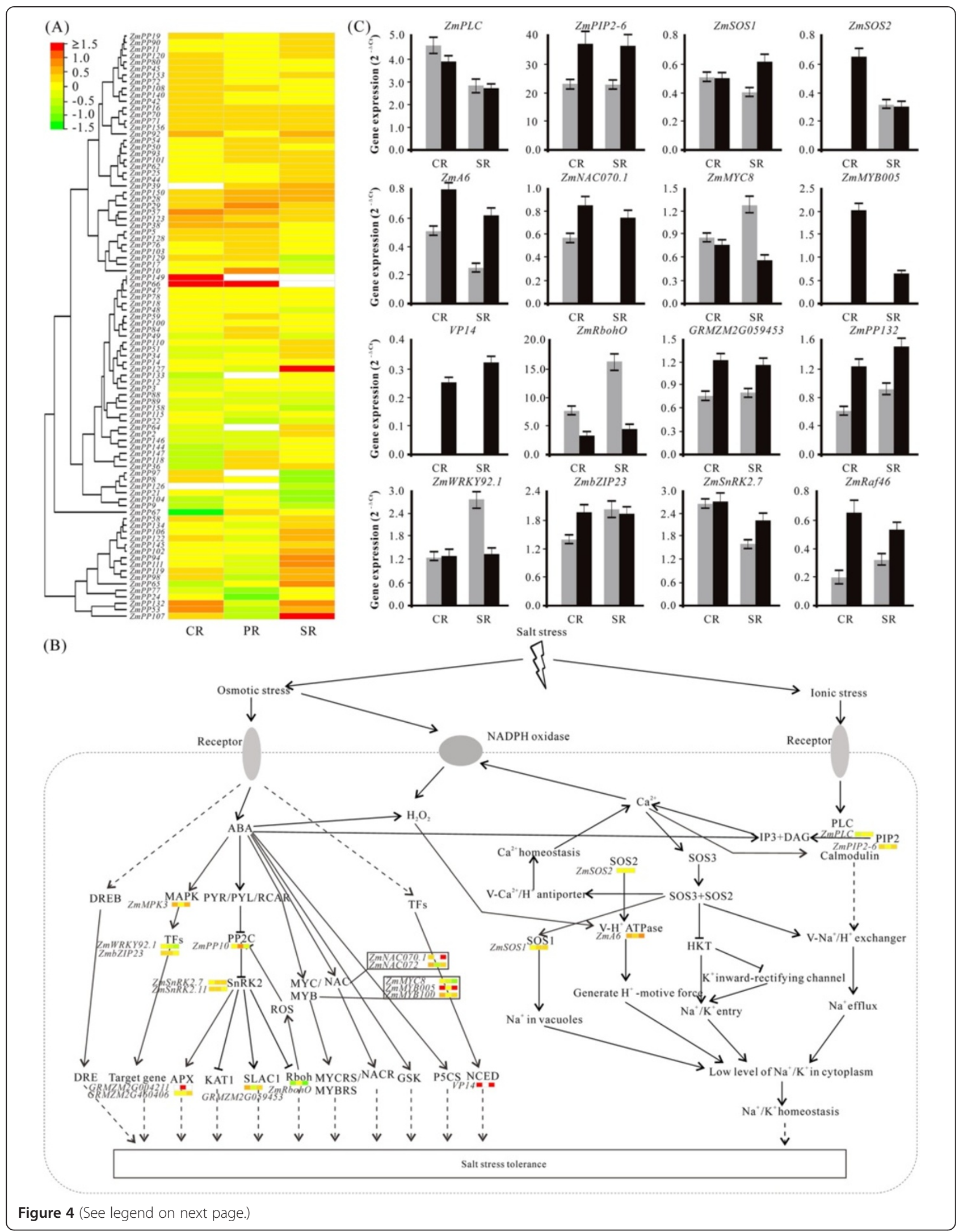


(See figure on previous page.)

Figure 4 Differential gene expression of ZmPP genes and overall signaling pathway under salt stress. A Expression profiles of ZmPPs under salt stress in crown root (CR), primary root (PR), and seminal root (SR). Log2 signal intensities were used to create the heatmap. White mean only expressed in control or have no expression value both in control and salt stress treatment. B Overview of salt-responsive systems in plants. C Real-time PCR results of salt-induced pathway components. Grey, control; black, salt treatment.

in Additional file 13. To detect whether all of these genes were implicated in salt signal transduction pathways, RNA-seq analysis of three types of roots of maize under salt treatment was performed (Additional file 14). As the partner of PPs, MAPK was reported to function significantly in salt signaling in yeast [51]. To investigate the role of MAPK cascades in maize salt stress, the expression patterns of MAPK pathway components in the three types of roots were analyzed. As shown in Additional file 15, we found that ZmRaf46 was expressed at relatively high level in SR and CR compared with PR, indicating that MAPK cascades might play crucial roles in salt stress response in maize. Apart from MAPK, SNF1-related protein kinases (SnRKs) which acted as the downstream elements of PPs, function in salt stress tolerance as well [52]. Among the 14 maize $S n R K 2 s, Z m S n R K 2.7$ and $Z m S n R K 2.11$ seem to play roles in salt stress tolerance with relative up-regulations (Additional file 14). Transcription factors (TFs) including MYB/MYC, NAC, WRKY, and zinc-finger protein have also been identified as salt stress-responsive factors in plants [50]. ZmWRKY33 (named ZmWRKY92.1 in our study), was reported to be significantly up-regulated in salt stress $[53,54]$. In contrast, the expression level of $Z m W R K Y 92.1$ has no obvious change in CR under salt stress, but is slightly down-regulated in PR and SR, indicating that $Z m W R K Y 92.1$ might act as a negative regulator of salt stress response. On the other hand, bZIP TFs function as ABA-dependent transcription factors and may be candidate genes enhancing crops stress tolerance [33,55]. With the result of RNA-seq analysis, ZmbZIP23 expression was up-regulated by salt treatment in maize CR and $\mathrm{PR}$, suggesting that ZmbZIP23 might play a partial role in response to salt stress. MYB TFs, which are characterized by highly conserved MYB DNA-binding domain, are involved in the regulation of salt stress response through ABA-independent pathway [50]. In this study, both ZmMYB005 and ZmMYB100 showed increased expressions in salinity, suggesting that they may act as master switch in the salt stress tolerance. NAC-type TFs also regulate some salt-responsive genes through ABA-dependent signaling pathway. In our analysis, high salinity stress induces several $Z m N A C s$ as well. It is notable that $Z m N A C 070.1$ and $Z m N A C 072$ showed significantly up-regulated expression in CR and SR. Additionally, plant exposure to high levels of $\mathrm{NaCl}$ also creates ionic stress in the form of cellular accumulation of $\mathrm{Cl}^{-}$and, in particular, $\mathrm{Na}^{+}$ions. Previous study showed that the vacuolar $\mathrm{Na}^{+} / \mathrm{H}^{+}$antiporter gene, $Z m N H X$ displayed an up-regulation in root when exposed to high $\mathrm{NaCl}$ concentration [50]. What's more, salt stress induce either influx or efflux of nutrient ions such as $\mathrm{Ca}^{2+}, \mathrm{K}^{+}$, and $\mathrm{NO}^{3-}$. The salt overly sensitive (SOS) signaling pathway that comprises SOS1, SOS2, and SOS3 is a well known signaling pathway for resistance to salt stress [50]. SOS2, a Ser/Thr PK, forms a complex with SOS3, an EF-hand $\mathrm{Ca}^{2+}$-binding protein, to activate SOS1, which plays a central role in sodium extrusion and controlling long distance $\mathrm{Na}^{+}$transport. Moreover, SOS2 also works to activate and interact with vacuolar $\mathrm{N}^{+} / \mathrm{H}^{+}$and $\mathrm{H}^{+} / \mathrm{Ca}^{2+}$ antiporters and V-ATPase, which will contribute to sequestration of excess $\mathrm{Na}^{+}$ions, further resulting in $\mathrm{Na}^{+}$ion homeostasis.

To confirm the expression patterns of these predicted genes under salt stress, 16 genes were selected for realtime PCR analysis. As shown in Figure $4 \mathrm{C}$, most of the selected genes were up-regulated in salt stress, while few were down-regulated. Among these 16 genes, ZmMYB005, $V P 14$, and ZmRaf46 were specially expressed under salt stress in both CR and SR, and ZmNAC070.1 displayed upregulation in $\mathrm{CR}$ and had specific expression in SR. Moreover, ZmSOS2 was expressed specially in CR but has no obvious fluctuation in SR. Taken together, real-time PCR-based expression profiling for these selected genes confirmed the outcome of RNA-seq analysis. Combined with RNA-seq, real-time PCR, and co-expression analysis (data not shown), ZmRaf46/49/17-ZmMKK3-ZmMPK3 might represent a potential MAPK cascade involved in salinity stress.

\section{Differential expression of ZmPP genes under drought stress}

It is well known that drought is a major environmental factor determining plant productivity and distribution. The previous researches suggested that some PP genes not only played crucial roles in stress-induced ABA accumulation but also function in redox signaling in maize $[19,21]$. PTP is a group of PP with 29 members in maize. To gain an insight into the functions of PTPs in ABA accumulation and stress responses, we examined the effect of PAO (a PTPase-specific inhibitor) [21] on the expression of the key enzymes involved in ABA biosynthesis, such as AAO3, NCED3, SDR1, ABA3, and ZEP in Arabidopsis. $\mathrm{ZmPP} 1$ is sensitive to the PAO and $50 \mu \mathrm{M}$ PAO was use to inactivated the enzyme. We found that PAO can arrest the expression of genes encoding the key enzymes (AAO3, NCED3, and $A B A 3)$ in the ABA biosynthesis pathway, indicating that PTPs may have the potential roles in ABA biosynthesis (Figure 5A). Li et al. 


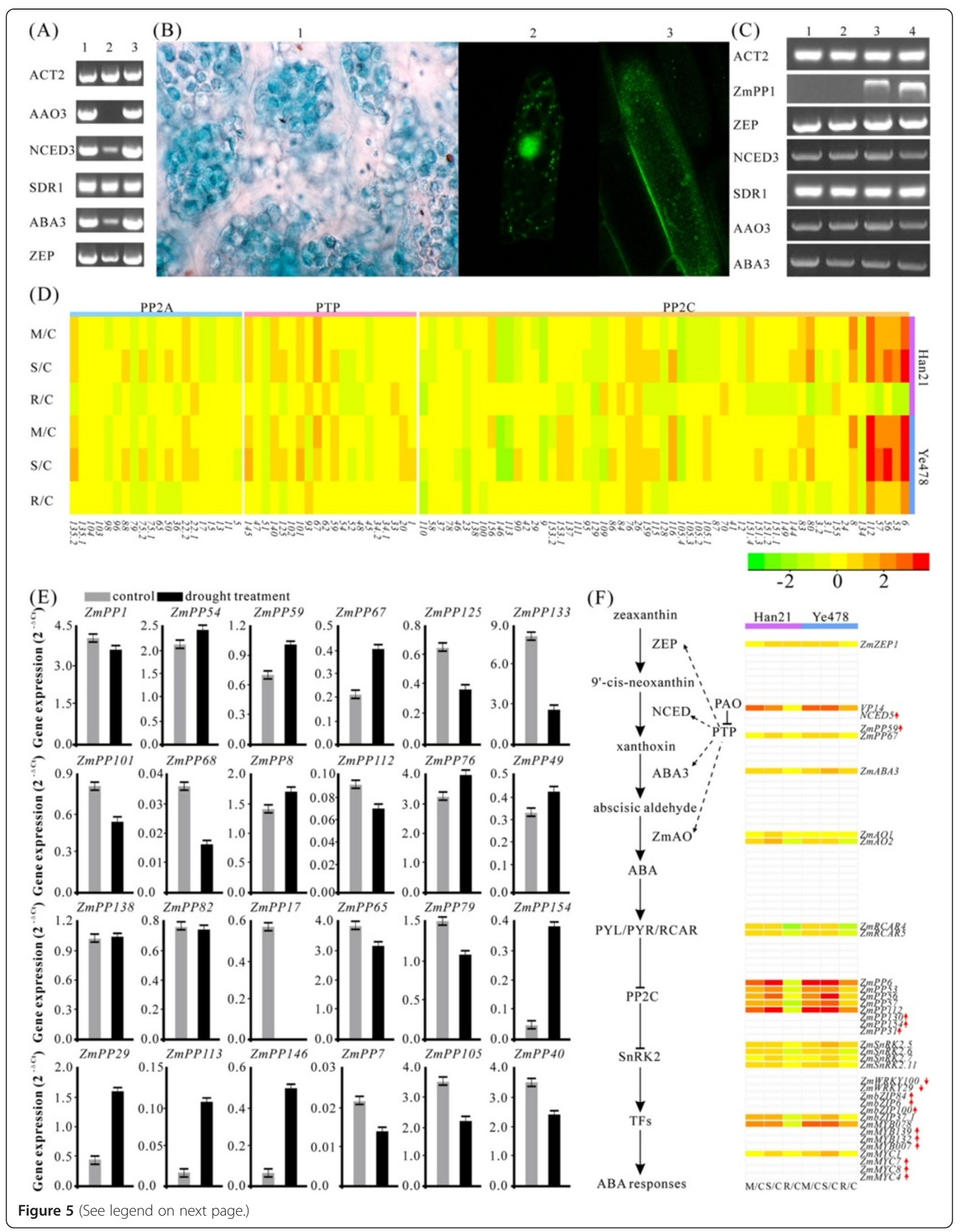


(See figure on previous page.)

Figure 5 Differential gene expression and real-time PCR analysis of ZmPP genes. A Effect of PAO on the key enzymes of ABA biosynthesis under water stress condition in Arabidopsis. 1, control; 2, PAO-treated; 3, negative control. B 1, stable expression of ZmPP1-GUS fusion protein in Arabidopsis chloroplast; 2, transient expression of ZmPP1-GFP fusion protein in onion epidermal cell; 3, transient expression of GFP protein only. C Analysis of gene expression in Arabidopsis transformed with estradiol-inducible ZmPP1. 1, wild-ethanol treated; 2, wild-estradiol treated; 3, ZmPP1 over-expression1 (ZmPP1OE1)-estradiol treated; ZmPP1OE2-estradiol treated. D Expression profiles of ZmPP genes under moderate drought stress (M/C), severe drought stress (S/C), and re-watering (R/C) as compared to control seedlings in Han21 and Ye478, respectively. E Real-time PCR analysis of 24 differently expressed PP genes under drought stress. $\mathbf{F}$ ABA biosynthesis and ABA-dependent pathway of response to drought in plants. The red arrows represent expression levels only detected by RNA-seq under drought stress.

has showed that ZmRIP1 (named ZmPP1 in this study), which is a member of PTPs, functioned in redox signaling in maize [21]. To better understand the cellular function of ZmPP1, we further examined its subcellular location (Figure 5B, Additional file 16). When GFPZmPP1 was introduced into onion epidermal cells, we found that $\mathrm{ZmPP} 1$ had multiple subcellular locations, whereas GFP alone was localized to the nucleus and cytoplasm (Figure 5B). Moreover, histochemical staining revealed that GUS expression occurred mainly in the chloroplasts. However, stable expression of ZmPP1 in Arabidopsis based on estrogen-receptor-based inducible system showed that ZmPP1 is not a mediator of stressinduced ABA accumulation (Figure $5 \mathrm{C}$ ).

To test whether the ZmPTPs play roles in drought stress, microarray data of different drought treatments were used to generate a heatmap. This genomic study of maize gene expression in response to water deficit include two different inbred lines, namely, drought-tolerant line Han21 and drought-sensitive line Ye478 (Figure 5D). Besides DNA microarray analysis, RNA-seq analysis was performed to investigate the functions of ZmPTPs in different drought sensitive organs, namely, leaf and cob, under drought and well-watered conditions [56]. Imposing the two-fold change requirement with a $P$ value $<0.05$, the public microarray data showed that expression of ZmPP67, a member of PTP subfamily, was up-regulated in Han21. Further observation from RNA-seq data showed that ZmPP67 was significantly up-regulated in both leaf and $\mathrm{cob}$, indicative of its important role in response to water deficit conditions. To confirm the expression patterns of ZmPTPs under water deficit condition, real-time PCR analysis was carried out using 8 differently expressed genes. As shown in Figure 5E, ZmPP67 was obviously upregulated in water deficient, which is consistent with our previous assumption that ZmPP67 might have an essential part during drought stress. Moreover, from RNA-seq and real-time PCR data, it was interesting to find that the other two PTP genes, ZmPP54 and ZmPP59, whose expression levels were also up-regulated. Of these 8 differentially expressed genes, 4 genes ( $Z m P P 125,-133,-101$ and -68) were down-regulated in water deficit condition. But the functions of these 4 genes in drought stress are still unknown.
Previous studies have reported that 6 Arabidopsis Group A PP2Cs interacted with ABA receptors and functioned as key negative regulators in ABA signaling [9,57]. Thus, in addition to PTPs, other PP genes may be involved in drought response. In RNA-seq data (Additional file 17), 10 genes (ZmPP6, -21, -29, -31, -113,-124,-127, -130,-146, and -154) were significantly up-regulated in leaf with the criterion that fold change $>2$. Of these 10 genes, all of them (except ZmPP124) were belong to PP2C class. After integrating the RNA-seq and real-time PCR data (Figure 5E, Additional file 17), it is notable that several putative PP orthologs among Arabidopsis, rice and maize showed strikingly consistent expression patterns, which lend further supporting to the existence of functional conservation among these species. For instance, $Z m P P 2 C$ (named ZmPP76), which was reported to be essential for plant stress signal transduction, showing up-regulated expression pattern in drought stress [19]. In our study, ZmPP76 was slightly up-regulated, whose expression pattern was in good agreement with the previous report [19]. Interestingly, another stress-induced gene $\mathrm{ZmPP} 2 \mathrm{Ca}$, named $Z m P P 8$ in this study, was upregulated by drought. In contrast, previous study has revealed that down-regulation of the $\mathrm{ZmPP} 2 \mathrm{Ca}$ transcript was observed in drought tolerant lines, whereas transcript levels were up-regulated in sensitive lines under drought stress [17]. In addition, $Z m P P 112$, the homolog of rice protein phosphatase OsPP2C1, was up-regulated more significantly in the drought sensitive line Ye478 than that in the drought tolerant line Han21. Previous study showed that OsPP2C1 was highly induced in response to abiotic stresses, even up-regulated at low temperature and drought conditions [58]. On the contrary, RNA-seq data and real-time PCR analyses of ZmPP112 exhibited decreased expression level in drought. Recently, Américo et al. reported that two members of Group A PP2C (ABI1 and PP2CA) can interact with the SnRK1 catalytic subunit, causing its dephosphorylation and inactivation, while the inhibition of PP2C allows ABA to promote SnRK1 activation [59]. $A B I 2$, the homolog of $A B I 1$ in Arabidopsis, has been reported to involve in ABA signal transduction with up-regulated expression pattern [60]. Similarly, ZmPP49, the homolog of Arabidopsis ABI2 in maize, was up-regulated, indicating that ZmPP49 might 
interact with $\mathrm{ABA}$ receptors to suppress the activity of SnRKs.

Apart from the above putative genes up-regulated by ABA, another two genes ( $Z m P P 138$ and $Z m P P 82)$ were expressed at nearly equivalent levels. As previously reported, the expression level of ZmPP138 (published $Z M P P 2)$ was relatively high in root and etiolated shoots [20]. Also, OsBIPP2C1, the homolog of ZmPP82 in rice, was up-regulated upon drought treatments [61]. In an attempt to examine the effects of drought stress on the expression of the PP2A genes, comprehensive analysis on microarray, RNA-seq and real-time PCR was done (Figure 5D-E, Additional file 17). Especially, ZmPP17 and $Z m P P 79$, two homologs of rice PP2As (OsPP2A-4 and $O s P P 2 A-2)$, showed special expression patterns. OsPP $2 A-4$ is expressed at comparably high levels in stems, roots and flowers, while an appreciably lower level of expression is observed in leaves. However, $\mathrm{ZmPP} 17$ was not expressed during drought in leaf [62]. Additionally, the expression level of OsPP $2 A-2$ was significantly downregulated in leaves and stems in response to drought stress, which is consistent with the expression pattern of ZmPP79 in our study [62]. Another PP2A gene, ZmPP65, showed down-regulated expression in leaf under drought stress. In addition to those genes that have been reported or had homologs in other species, some maize PP genes were also found to be up-regulated under water deficit, such as ZmPP29, $-113,-146$ and -154 . Typically, $Z m P P 154$ is a member of Group A PP2C in maize. In addition, $Z m P P 7, Z m P P 105$ and $Z m P P 40$ were especially down-regulated under water deficit condition.

A putative schematic of the ABA biosynthesis and ABA cellular response during drought stress was proposed based on the described web-like networks of ABA biosynthesis and ABA signaling under water shortage condition (Figure 5F). Compared with Arabidopsis, maize has less characterized drought-induced genes (Additional files 18 and 19). Based on our investigation data and the previous research results $[63,64]$, we found that ABA biosynthetase genes were up-regulated in the drought-tolerant line Han21, although their protein levels have not been examined in most cases.

\section{Expression profiling of ZmPP genes under cold stress}

Cold responses have been observed in many plants, like Arabidopsis and rice, which alter mRNA levels of genes belonging to multiple independent pathways to response to low temperature $[65,66]$. To search for the molecular mechanism of high intraspecific diversity in cold tolerance of maize, transcriptomic analysis was studied in two inbred lines contrasting in chilling tolerance (Additional file 20). With the criterion that the response must be at least 2.8 -fold (|log2[(expression level in the cold):(expression level in control conditions)] $\mid \geq 1.5)[11], 13 \mathrm{ZmPP}$ genes
(ZmPP6, -24,-29, -66, -77, -82, -92, -112, -116, $-127,-149,-154$, and -155$)$ were found to be significantly up-regulated, while three genes (ZmPP69, -87, and -101) displayed obvious down-regulation. AtPP2CA, an Arabidopsis group A PP2C, was reported as a negative regulator in ABA responses during cold acclimation [12]. ZmPP6, the homolog of AtPP2CA in maize, which was upregulated in cold-tolerant line ETH-DH7. In addition, ZmPP2C2, named ZmPP159 in this study, occurred to be a positive regulator of cold resistance in plant [18], whose expression was slightly up-regulated. It is conceivable that maize PP2C activity could be modulated via cold stressinduced response. To investigate the role of MAPK cascades in cold tolerance, we performed microarray analysis of components of MAPK pathway (Additional file 21). In cold tolerant line, three MAPKs (ZmMPK6, -8 and -12), two MAPKKs (ZmMKK2 and ZmMKK4) and four MP3Ks (ZmMAPKKK1, ZmMAPKKK17, ZmZIK9 and ZmRaf43) were significantly up-regulated. To explore the gene expression patterns in our maize inbred lines under cold stress, the obviously up-regulated $Z m P P s$ and the components of MAPK cascades were selected for real-time PCR analysis. As shown in Additional file 22, all the selected genes were up-regulated under cold treatment. We proposed that MAP3K1/17/ZIK9/Raf43-MKK2/4-MAPK6/8/ 12 might represent putative MAPKK-MAPK or MAPKKKMAPKK-MAPK cascades based on microarray, real-time PCR and co-expression analyses (data not shown). The predicted MAPKKK-MAPKK-MAPK phosphorylation regulatory network constitutes a valuable resource to understand the function and specificity of MAPK signaling systems in maize. It is worth mentioning that ZmMP3K1and ZmMPK12-overexpression in tobacco showed higher seed germination rate under low temperature treatment $\left(12^{\circ} \mathrm{C}\right)$ (data not shown).

\section{Promoter analysis and miRNA targets of ZmPPs}

In genetics, a promoter is a region of DNA that initiates transcription of a particular gene and confers developmental and/or environmental regulation of gene expression. Discovery of cis-regulatory elements in the promoter regions is essential to understand the spatial and temporal expression pattern of $\mathrm{ZmPP}$ genes. To further expound the transcription regulation machineries of the $159 \mathrm{ZmPP}$ genes in maize, a comprehensive promoter analysis was performed by PlantCARE database [67]. The occurrence of cis-elements in ZmPP genes are showed in Additional file 23 . The statistical analysis showed that 12 cis-elements were enriched in maize PP genes, which were involved in regulation of gene expression under stress conditions. Nearly all genes (except $Z m P P 13,-71,-125)$ contained part of these 12 cis-elements, and the elements were variably present in the promoter regions of most $\mathrm{ZmPP}$ genes. Interestingly, we found that the majority of the $\mathrm{ZmPP}$ 
genes contained ABRE, G-Box and MBS, which are the TF binding sites for bZIP, GBF (G-Box-binding factor) and MYB, respectively. The data might indicate cis-elements in this study may play major roles in regulating the expression of PP genes in response to different stresses in maize.

MicroRNAs (miRNAs) play important roles in plant post-transcriptional gene regulation by either cleaving mRNA transcripts or repressing protein translation [68]. Putative miRNAs targeting the ZmPP genes were identified using Target-align program. It showed that about 35 $\mathrm{ZmPP}$ genes were targeted by maize miRNAs (Additional file 24). In addition to regulating growth and development, most miRNAs in plants play important roles in the regulation of various cellular processes underlying plant adaptation to environmental stresses. Among these target genes, 6 and 8 members belong to Group A PP2C and PP2A, respectively, indicating that miRNAs in the present study would assist in understanding the post-transcriptional control of gene regulation during physiological and stressinduced cellular responses.

\section{Conclusions}

Conclusively, our results present a comprehensive account of PP-encoding genes and provide new insights into the phylogenetic relationships and characteristic functions of maize PPs. In addition, analyses of expression profiles based on microarray and RNA-seq method unraveled their probable functions during different developmental stages and abiotic stresses and will be useful in studies aimed at revealing the global regulatory network in maize development and abiotic stress responses, thereby contributing to the maize molecular breeding with enhanced quality traits.

\section{Additional files}

Additional file 1: Figure S1. Phylogenetic analysis of maize, rice, and Arabidopsis PP genes. An un-rooted NJ tree is made based on the catalytic domain sequences of maize, rice, and Arabidopsis PPs. PPs from Arabidopsis, rice, and maize belong to the same class falling in the same clades. Scale bar represents 0.1 amino acid substitutions per site.

Additional file 2: Figure S2. The map of intron/exon arrangement of ZmPP genes.

Additional file 3: Figure S3. Circos diagram of protein phosphatase gene pairs between maize and rice genomes. Outer two circles Distribution of each of the PP genes and scaled chromosomes for each species in million bp (Mb) units, respectively. Histograms below each chromosome Number of introns of PK genes: green $<5$ introns, red $\geq 10$ introns. Boxes Syntenic regions. Colors are assigned to the syntenic regions according to the colors of the corresponding chromosome. Innermost colored lines interconnect putative orthologous PP gene pairs between rice and maize.

Additional file 4: Figure S4. Heatmap showing the clustering of ZmPPs according to their expression profiles of 60 detected transcripts at different stages/organs of maize. Red, white and green indicate high, medium and low levels of gene expression, respectively. E2enzyme was used as a internal control.
Additional file 5: Figure S5. A putative schematic of root development signaling pathway in maize. The little colored blocks besides the gene expression level of pathway components under different developmental stages.

Additional file 6: Figure S6. RNA-seq differentially expressed analysis of ZmPP genes along maize leaf developmental gradients, namely, base, $-1 \mathrm{~cm},+4 \mathrm{~cm}$, and tip. E2enzyme was used as a internal control.

Additional file 7: Figure S7. Expression patterns of ZmPP genes in 12 diverse maize reproductive tissues. DAP, days after pollination; Pre-em, preemergence; Post-em, postemergence. E2enzyme was used as a internal control.

Additional file 8: Figure S8. Expression profiles of signaling components under salt stress in CR, PR, and SR, respectively. Log2 based fold changes were used to create the heatmap. Expression values highlight with white mean only expressed in control or have no expression value both in control and salt stress treatment.

Additional file 9: Figure S9. The vector map of pEZS-NL-ZmPP1. Additional file 10: Figure S10. Real-time PCR analysis of representative ZmPPs and MAPK-cascade genes under cold treatments. The maize ACTIN7 gene was used as endogenous control to normalize data. E2 enzyme was used as a internal control.

Additional file 11: Table S1. List of primers used in this study. Additional file 12: Table S2. The identified maize protein phosphatases and their related information.

Additional file 13: Table S3. List of 152 detected ZmPP genes and their expression patterns at 18 selected tissues.

Additional file 14: Table S4. List of putative root development signaling components in maize.

Additional file 15: Table S5. List of flowering time signaling components in maize.

Additional file 16: Table S6. List of putative salt stress signaling components in maize.

Additional file 17: Table S7. List of expression values of MAPK cascade-related genes and key genes of ABA biosynthesis in maize under salt stress.

Additional file 18: Table S8. List of FPKM values of ZmPPs in maize reproductive (cob) and vegetative tissue (leaf) under both drought and well-watered conditions. MCC and MCD stand for maize ovary, well watered and drought, respectively; MLC and MLD stand for maize basal leaf meristem, well watered and drought, respectively. Numbers 1 and 2 indicate the two biological replicates. The extent of differential expression is measured in terms of fold change and (-) indicates failure to calculate or undetected values. Values in red and blue indicate the fold increase and decrease in expression in the drought-stressed tissue, respectively.

Additional file 19: Table S9. List of FPKM values of components involved in $A B A$ biosynthesis and ABA-dependent pathway of response to drought in vegetative tissue (leaf) under both drought and well-watered conditions. MLC and MLD stand for maize basal leaf meristem, well watered and drought, respectively. Numbers 1 and 2 indicate the two biological replicates. The extent of differential expression is measured in terms of fold change and (-) indicates failure to calculate or undetected values. Values in red and blue indicate the fold increase and decrease in expression in the drought-stressed tissue, respectively.

Additional file 20: Table S10. List of putative components involved in ABA biosynthesis and ABA-dependent pathway of response to drought in maize.

Additional file 21: Table S11. List of expression values of ZmPP genes under cold stress. ETH-DH7 log(c), ETH-DH7 log(k), ETH-DL3 log(c), ETH-DL3 log(k) means intensity (AU) of fluorescence of labeled aaRNA from cold-treatment (c) or control ( $k$ ) hybridizing to the probe, shown as $\log 2$ in the respective maize line. DH7 $\log (\mathrm{c} / \mathrm{k}), \mathrm{DL} 3 \log (\mathrm{c} / \mathrm{k})$ means $\log 2$ of ratio of expression in cold-treated vs. control of the probe in the respective maize line, respectively. Values in red and blue indicate the fold increase and decrease in expression in the drought-stressed tissue, respectively. 
Additional file 22: Table S12. List of expression values of MAPK cascades genes in cold stress. ETH-DH7 log(c), ETH-DH7 log(k), ETH-DL3 $\log (\mathrm{c}), \mathrm{ETH}-\mathrm{DL} 3 \log (\mathrm{k})$ means intensity (AU) of fluorescence of labeled aaRNA from cold-treatment (c) or control ( $k$ ) hybridizing to the probe, shown as $\log 2$ in the respective maize line. ETH-DH7 $\log (\mathrm{c} / \mathrm{k}), \mathrm{ETH}-\mathrm{DL} 3$ $\log (\mathrm{c} / \mathrm{k})$ means $\log 2$ of ratio of expression in cold-treated vs. control of the probe in the respective maize line, respectively.

Additional file 23: Table S13. List of promoters in ZmPP genes. Additional file 24: Table S14. List of predicted miRNA-regulated ZmPP genes.

\section{Competing interests}

The authors declare that they have no competing interests.

\section{Authors' contributions}

KW conceptualized, designed the project. SP carried out computational analysis, microarray and RNA-seq expression profiling. KW and SP performed the experiments. SP and KW wrote the manuscript. Both authors read and approved the final manuscript.

\section{Acknowledgments}

We are grateful to the providers who submitted the microarray and RNA-seq data to the public expression databases which can be applied freely. We are thankful to Prof. Wensuo Jia in China Agriculture University for providing us guidance during the project work. We sincerely thank Dr. Yanmei Wang in Tshinghua University for revising our manuscript critically. We also thank Yanhui Chen, Yina Lin, Xiaojun Zhong, and Xiaoyao Liu in our laboratory for useful discussions.

Received: 13 June 2014 Accepted: 3 September 2014

Published: 9 September 2014

\section{References}

1. Luan S: Protein phosphatases in plants. Annu Rev Plant Biol 2003, 54:63-92.

2. Wei KF, Wang YM, Xie DX: Identification and expression profile analysis of the protein kinase gene superfamily in maize development. Mol Breeding 2014, 33(1):155-172.

3. Wei KF, Wang YM, Zhong XJ, Pan S: Protein kinase structure, expression and regulation in maize drought signaling. Mol Breeding 2014, 34(2):583-602.

4. Kwon YG, Lee SY, Choi Y, Greengard P, Nairn AC: Cell cycle-dependent phosphorylation of mammalian protein phosphatase 1 by cdc2 kinase. Proc Natl Acad Sci U S A 1997, 94(6):2168-2173.

5. Zhou HW, Nussbaumer C, Chao Y, DeLong A: Disparate roles for the regulatory A subunit isoforms in Arabidopsis protein phosphatase $2 \mathrm{~A}$. Plant Cell 2004, 16(3):709-722.

6. Song SK, Clark SE: POL and related phosphatases are dosage-sensitive regulators of meristem and organ development in Arabidopsis. Dev Biol 2005, 285(1):272-284.

7. Kim DH, Kang JG, Yang SS, Chung KS, Song PS, Park CM: A phytochromeassociated protein phosphatase $2 \mathrm{~A}$ modulates light signals in flowering time control in Arabidopsis. Plant Cell 2002, 14(12):3043-3056.

8. Zhang Q: Strategies for developing green super rice. Proc Natl Acad Sci U S A 2007, 104(42):16402-16409.

9. Komatsu K, Suzuki N, Kuwamura M, Nishikawa Y, Nakatani M, Ohtawa H, Takezawa D, Seki M, Tanaka M, Taji T, Hayashi T, Sakata Y: Group A PP2Cs evolved in land plants as key regulators of intrinsic desiccation tolerance. Nat Commun 2013, 4:2219.

10. Liu X, Zhu Y, Zhai H, Cai H, Ji W, Luo X, Li J, Bai X: AtPP2CG1, a protein phosphatase $2 \mathrm{C}$, positively regulates salt tolerance of Arabidopsis in abscisic acid-dependent manner. Biochem Biophys Res Commun 2012, 422(4):710-715.

11. Sobkowiak A, Jonczyk M, Jarochowska E, Biecek P, Trzcinska-Danielewicz J, Leipner J, Fronk J, Sowinski P: Genome-wide transcriptomic analysis of response to low temperature reveals candidate genes determining divergent cold-sensitivity of maize inbred lines. Plant Mol Biol 2014, 85(3):317-331

12. Tahtiharju S, Palva T: Antisense inhibition of protein phosphatase $2 \mathrm{C}$ accelerates cold acclimation in Arabidopsis thaliana. Plant J 2001, 26(4):461-470
13. Kerk D, Bulgrien J, Smith DW, Barsam B, Veretnik S, Gribskov M: The complement of protein phosphatase catalytic subunits encoded in the genome of Arabidopsis. Plant Physiol 2002, 129(2):908-925.

14. Singh A, Giri J, Kapoor S, Tyagi AK, Pandey GK: Protein phosphatase complement in rice: genome-wide identification and transcriptional analysis under abiotic stress conditions and reproductive development. BMC Genomics 2010, 11:435.

15. Schweighofer A, Hirt H, Meskiene I: Plant PP2C phosphatases: emerging functions in stress signaling. Trends Plant Sci 2004, 9(5):236-243.

16. Sommer D, Wells TA, Song PS: A possible tyrosine phosphorylation of phytochrome. FEBS Lett 1996, 393(2-3):161-166.

17. Li FH, Fu FL, Sha FL, He L, Li WC: Differential expression of serine/ threonine protein phosphatase type-2C under drought stress in maize. Plant Mol Biol Rep 2008, 27(1):29-37.

18. Hu X, Liu L, Xiao B, Li D, Xing X, Kong X: Enhanced tolerance to low temperature in tobacco by over-expression of a new maize protein phosphatase 2C, ZmPP2C2. J Plant Physiol 2010, 167(15):1307-1315.

19. Liu L, Hu X, Song J, Zong X, Li D: Over-expression of a Zea mays L. protein phosphatase $2 \mathrm{C}$ gene (ZmPP2C) in Arabidopsis thaliana decreases tolerance to salt and drought. J Plant Physiol 2009, 166(5):531-542.

20. Broz AK, Thelen JJ, Muszynski MG, Miernyk JA, Randall DD: ZMPP2, a novel type-2C protein phosphatase from maize. J Exp Bot 2001, 52(361):1739-1740.

21. Li B, Zhao Y, Liang L, Ren $H$, Xing $Y$, Chen L, Sun M, Wang Y, Han Y, Jia H, Huang C, Jia W: Purification and characterization of ZmRIP1, a novel reductant-inhibited protein tyrosine phosphatase from maize. Plant Physiol 2012, 159(2):671-681

22. Smith $\mathrm{RD}$, Walker JC: Isolation and expression of a maize type 1 protein phosphatase. Plant Physiol 1991, 97(2):677-683.

23. Finn RD, Clements J, Eddy SR: HMMER web server: interactive sequence similarity searching. Nucleic Acids Res 2011, 39(Web Server issue):W29-W37.

24. Soderlund C, Bomhoff M, Nelson WM: SyMAP v3.4: a turnkey synteny system with application to plant genomes. Nucleic Acids Res 2011, 39(10):e68

25. Rozas J, Sanchez-DelBarrio JC, Messeguer X, Rozas R: DnaSP, DNA polymorphism analyses by the coalescent and other methods. Bioinformatics 2003, 19(18):2496-2497.

26. Yang Z, Gu S, Wang X, Li W, Tang Z, Xu C: Molecular evolution of the CPP-like gene family in plants: insights from comparative genomics of Arabidopsis and rice. J Mol Evol 2008, 67(3):266-277.

27. Wei KF, Chen YH, Lin YN, Pan YT: Genetic dynamic analysis of the influenza A H5N1 NS1 gene in China. PLoS One 2014, 9(7):e101384.

28. Zuo J, Niu QW, Chua NH: Technical advance: An estrogen receptor-based transactivator XVE mediates highly inducible gene expression in transgenic plants. Plant J 2000, 24(2):265-273.

29. Xie F, Zhang B: Target-align: a tool for plant microRNA target identification. Bioinformatics 2010, 26(23):3002-3003.

30. Griffiths-Jones S, Grocock RJ, van Dongen S, Bateman A, Enright AJ: miRBase: microRNA sequences, targets and gene nomenclature. Nucleic Acids Res 2006, 34(Database issue):D140-D144.

31. Xue T, Wang D, Zhang S, Ehlting J, Ni F, Jakab S, Zheng C, Zhong Y: Genome-wide and expression analysis of protein phosphatase $2 \mathrm{C}$ in rice and Arabidopsis. BMC Genomics 2008, 9:550

32. Manning $G$, Whyte DB, Martinez R, Hunter T, Sudarsanam $S$ : The protein kinase complement of the human genome. Science 2002, 298(5600):1912-1934.

33. Wei K, Chen J, Wang Y, Chen Y, Chen S, Lin Y, Pan S, Zhong X, Xie D: Genome-wide analysis of bZIP-encoding genes in maize. DNA Res 2012, 19(6):463-476

34. Zhang Z, Kishino H: Genomic background predicts the fate of duplicated genes: evidence from the yeast genome. Genetics 2004, 166(4):1995-1999.

35. Hanada K, Zou C, Lehti-Shiu MD, Shinozaki K, Shiu SH: Importance of lineage-specific expansion of plant tandem duplicates in the adaptive response to environmental stimuli. Plant Physiol 2008, 148(2):993-1003.

36. Zhou S, Wei F, Nguyen J, Bechner M, Potamousis K, Goldstein S, Pape L, Mehan MR, Churas C, Pasternak S, Forrest DK, Wise R, Ware D, Wing RA, Waterman MS, Livny M, Schwartz DC: A single molecule scaffold for the maize genome. PLoS Genet 2009, 5(11):e1000711.

37. Cannon SB, Mitra A, Baumgarten A, Young ND, May G: The roles of segmental and tandem gene duplication in the evolution of large gene families in Arabidopsis thaliana. BMC Plant Biol 2004, 4:10.

38. Wei KF, Chen YH, Xie DX: Genome-scale evolution and phylodynamics of H5N1 influenza virus in China during 1996-2012. Vet Microbiol 2013, 167(3-4):383-393. 
39. Wei KF, Lin YN, Xie DX: Evolutionary and ecological dynamics of transboundary disease caused by $\mathrm{H} 5 \mathrm{~N} 1$ virus in southeast Asia. Transbound Emerg Dis 2013, doi:10.1111/tbed.12147.

40. Williams RW, Wilson JM, Meyerowitz EM: A possible role for kinaseassociated protein phosphatase in the Arabidopsis CLAVATA1 signaling pathway. Proc Natl Acad Sci U S A 1997, 94(19):10467-10472.

41. Wei KF, Chen J, Chen YF, Wu $\sqcup$, Xie DX: Molecular phylogenetic and expression analysis of the complete WRKY transcription factor family in maize. DNA Res 2012, 19(2):153-164.

42. Hoecker N, Keller B, Piepho HP, Hochholdinger F: Manifestation of heterosis during early maize (Zea mays L.) root development. Theor App/ Genet 2006, 112(3):421-429.

43. Michniewicz M, Zago MK, Abas L, Weijers D, Schweighofer A, Meskiene I, Heisler MG, Ohno C, Zhang J, Huang F, Schwab R, Weigel D, Meverowitz EM, Lushnig C, Offringa R, Friml J: Antagonistic regulation of PIN phosphorylation by PP2A and PINOID directs auxin flux. Cell 2007, 130(6):1044-1056

44. Forestan C, Farinati S, Varotto S: The maize PIN gene family of auxin transporters. Front Plant Sci 2012, 3:16.

45. Li P, Ponnala L, Gandotra N, Wang L, Si Y, Tausta SL, Kebrom TH, Provart N, Patel R, Myers CR, Reidel EJ, Turgeon R, Liu P, Sun Q, Nelson T, Brutnell TP: The developmental dynamics of the maize leaf transcriptome. Nat Genet 2010, 42(12):1060-1067.

46. Dong Z, Danilevskaya O, Abadie T, Messina C, Coles N, Cooper M: A gene regulatory network model for floral transition of the shoot apex in maize and its dynamic modeling. PLoS One 2012, 7(8):e43450.

47. Fan C, Hu R, Zhang X, Wang X, Zhang W, Zhang Q, Ma J, Fu YF: Conserved CO-FT regulons contribute to the photoperiod flowering control in soybean. BMC Plant Biol 2014, 14:9.

48. Markelz NH, Costich DE, Brutnell TP: Photomorphogenic responses in maize seedling development. Plant Physiol 2003, 133(4):1578-1591.

49. Jeong S, Clark SE: Photoperiod regulates flower meristem development in Arabidopsis thaliana. Genetics 2005, 169(2):907-915.

50. Kumar K, Kumar M, Kim SR, Ryu H, Cho YG: Insights into genomics of salt stress response in rice. Rice (N Y) 2013, 6(1):27.

51. Kumar K, Sinha AK: Overexpression of constitutively active mitogen activated protein kinase kinase 6 enhances tolerance to salt stress in rice. Rice (N Y) 2013, 6(1):25.

52. Kulik A, Wawer I, Krzywinska E, Bucholc M, Dobrowolska G: SnRK2 protein kinases-key regulators of plant response to abiotic stresses. OMICS 2011, 15(12):859-872.

53. Wei K, Chen J, Chen Y, Wu L, Xie D: Multiple-strategy analyses of ZmWRKY subgroups and functional exploration of ZmWRKY genes in pathogen responses. Mol Biosyst 2012, 8(7):1940-1949.

54. Shekhawat UK, Ganapathi TR: MusaWRKY71 overexpression in banana plants leads to altered abiotic and biotic stress responses. PLoS One 2013, 8(10):e75506.

55. Ying S, Zhang DF, Fu J, Shi YS, Song YC, Wang TY, Li Y: Cloning and characterization of a maize bZIP transcription factor, ZmbZIP72, confers drought and salt tolerance in transgenic Arabidopsis. Planta 2012, 235(2):253-266.

56. Kakumanu A, Ambavaram MM, Klumas C, Krishnan A, Batlang U, Myers E, Grene R, Pereira A: Effects of drought on gene expression in maize reproductive and leaf meristem tissue revealed by RNA-Seq. Plant Physiol 2012, 160(2):846-867.

57. Bhaskara GB, Nguyen $\Pi$, Verslues PE: Unique drought resistance functions of the highly ABA-induced clade A protein phosphatase 2 Cs. Plant Physiol 2012, 160(1):379-395.

58. Jiang $Y Y$, Dong JL, Chen RJ, Gao XL, Xu ZJ: Isolation of a novel PP2C gene from rice and its response to abiotic stresses. Afr J Biotechnol 2011, 10(37):7143-7154.

59. Rodrigues A, Adamo M, Crozet P, Margalha L, Confraria A, Martinho C, Elias A, Rabissi A, Lumbreras V, Gonzalez-Guzman M, Antoni R, Rodriguez PL, Baena Gonzalez E: ABI1 and PP2CA phosphatases are negative regulators of Snf1-related protein kinase1 signaling in Arabidopsis. Plant Cell 2013, 25(10):3871-3884

60. Rodriguez PL, Benning G, Grill E: $A B I 2$, a second protein phosphatase $2 C$ involved in abscisic acid signal transduction in Arabidopsis. FEBS Lett 1998, 421(3):185-190.
61. Zhang XC, Yu X, Zhang HJ, Song FM: Molecular characterization of a defense-related AMP-binding protein gene, OSBIABP1, from rice. J Zhejiang Univ Sci B 2009, 10(10):731-739.

62. Yu RM, Wong MM, Jack RW, Kong RY: Structure, evolution and expression of a second subfamily of protein phosphatase $2 \mathrm{~A}$ catalytic subunit genes in the rice plant (Oryza sativa L.). Planta 2005, 222(5):757-768.

63. Capelle V, Remoue C, Moreau L, Reyss A, Mahe A, Massonneau A, Falque M, Charcosset A, Thevenot C, Rogowsky P, Coursol S, Prioul JL: QTLs and candidate genes for desiccation and abscisic acid content in maize kernels. BMC Plant Biol 2010, 10:2.

64. Vallabhaneni R, Bradbury LM, Wurtzel ET: The carotenoid dioxygenase gene family in maize, sorghum, and rice. Arch Biochem Biophys 2010, 504(1):104-111.

65. Kreps JA, Wu Y, Chang HS, Zhu T, Wang X, Harper JF: Transcriptome changes for Arabidopsis in response to salt, osmotic, and cold stress. Plant Physiol 2002, 130(4):2129-2141.

66. Cheng C, Yun KY, Ressom HW, Mohanty B, Bajic VB, Jia Y, Yun SJ, de los Reyes BG: An early response regulatory cluster induced by low temperature and hydrogen peroxide in seedlings of chilling-tolerant japonica rice. BMC Genomics 2007, 8:175.

67. Lescot M, Dehais $P$, Thijs $G$, Marchal $K$, Moreau $Y$, Van de Peer $Y$, Rouze $P$, Rombauts S: PlantCARE, a database of plant cis-acting regulatory elements and a portal to tools for in silico analysis of promoter sequences. Nucleic Acids Res 2002, 30(1):325-327.

68. Wei KF, Wu L, Chen J, Chen YF, Xie DX: Structural evolution and functional diversification analyses of argonaute protein. $J$ Cell Biochem 2012, 113(8):2576-2585.

doi:10.1186/1471-2164-15-773

Cite this article as: Wei and Pan: Maize protein phosphatase gene family: identification and molecular characterization. BMC Genomics 2014 15:773.

\section{Submit your next manuscript to BioMed Central and take full advantage of:}

- Convenient online submission

- Thorough peer review

- No space constraints or color figure charges

- Immediate publication on acceptance

- Inclusion in PubMed, CAS, Scopus and Google Scholar

- Research which is freely available for redistribution 